Draft Version July 1, 2021

Typeset using LATEX twocolumn style in AASTeX63

\title{
Resolving the circumstellar environment of the Galactic B[e] supergiant star MWC 137. II. Nebular kinematics and stellar variability*
}

\author{
Michaela Kraus, ${ }^{1}$ Tinna Limmets, ${ }^{1,2}$ Alexei Moiseev ${ }^{3}$ Julieta P. Sánchez Arias, ${ }^{1}$ Dieter H. Nickeler, ${ }^{1}$ \\ Lydia S. Cidale, ${ }^{4,5}$ And David Jones ${ }^{6,7}$ \\ ${ }^{1}$ Astronomical Institute, Czech Academy of Sciences, 25165 Ondřejov, Czech Republic \\ 2 Tartu Observatory, University of Tartu, Observatooriumi 1, 61602 Tóravere, Estonia \\ ${ }^{3}$ Special Astrophysical Observatory, Russian Academy of Sciences, Nizhnii Arkhyz 369167, Russia \\ ${ }^{4}$ Departamento de Espectroscopía, Facultad de Ciencias Astronómicas y Geofísicas, Universidad Nacional de La Plata, Paseo del Bosque \\ S/N, La Plata, B1900FWA, Buenos Aires, Argentina \\ ${ }^{5}$ Instituto de Astrofísica de La Plata (CCT La Plata-CONICET, UNLP), Paseo del Bosque S/N, La Plata, B1900FWA, Buenos Aires, \\ Argentina \\ ${ }^{6}$ Instituto de Astrofísica de Canarias, E-38205 La Laguna, Tenerife, Spain \\ ${ }^{7}$ Departamento de Astrofísica, Universidad de La Laguna, E-38206 La Laguna, Tenerife, Spain
}

\begin{abstract}
The Galactic B[e] supergiant MWC 137 is surrounded by a large-scale optical nebula. To shed light on the physical conditions and kinematics of the nebula, we analyze the optical forbidden emission lines [N II] $\lambda \lambda 6548,6583$ and [S II] $\lambda \lambda 6716,6731$ in long-slit spectra taken with ALFOSC at the Nordic Optical Telescope. The radial velocities display a complex behavior but, in general, the northern nebular features are predominantly approaching while the southern ones are mostly receding. The electron density shows strong variations across the nebula with values spreading from about zero to $\sim 800 \mathrm{~cm}^{-3}$. Higher densities are found closer to MWC 137 and in regions of intense emission, whereas in regions with high radial velocities the density decreases significantly. We also observe the entire nebula in the two [S II] lines with the scanning Fabry-Perot interferometer attached to the 6-m telescope of the Special Astrophysical Observatory. These data reveal a new bow-shaped feature at $\mathrm{PA}=225-245^{\circ}$ and a distance $80^{\prime \prime}$ from MWC 137. A new $\mathrm{H} \alpha$ image has been taken with the Danish 1.54-m telescope on La Silla. No expansion or changes in the nebular morphology appear within 18.1 years. We derive a mass of $37_{-5}^{+9} M_{\odot}$ and an age of $4.7 \pm 0.8 \mathrm{Myr}$ for MWC 137. Furthermore, we detect a period of $1.93 \mathrm{~d}$ in the time series photometry collected with the TESS satellite, which could suggest stellar pulsations. Other, low-frequency variability is seen as well. Whether these signals are caused by internal gravity waves in the early-type star or by variability in the wind and circumstellar matter currently cannot be distinguished.
\end{abstract}

Keywords: circumstellar matter — stars: early-type — stars: massive — stars: individual (MWC 137) - supergiants

\section{INTRODUCTION}

Corresponding author: Michaela Kraus

michaela.kraus@asu.cas.cz

* Based on observations collected with 1) the Nordic Optical Telescope, operated by the Nordic Optical Telescope Scientific Association at the Observatorio del Roque de los Muchachos, La Palma, Spain, of the Instituto de Astrofisica de Canarias, 2) the Danish 1.54-m telescope at La Silla, Chile, and 3) the 6-m telescope of the Special Astrophysical Observatory (SAO), Russia.
The class of $\mathrm{B}[\mathrm{e}]$ supergiants $(\mathrm{B}[\mathrm{e}] \mathrm{SG})$ consists of evolved, luminous massive stars, which display indications of a circumstellar dusty disk and a bipolar linedriven wind (Zickgraf et al. 1985; Lamers et al. 1998). Their main optical spectral characteristics are intense and broad Balmer line emission along with narrow emission lines of low-ionized and neutral metals of both permitted and forbidden transitions, in particular [O I]. Moreover, many B[e]SGs display intense emission of the Ca II infrared triplet lines and of [CaII] (Aret et al. 2012; Kraus 2019). These optical forbidden emission lines have been identified as valuable tracers for the cir- 
cumstellar gas disks of $\mathrm{B}[\mathrm{e}] \mathrm{SGs}$ and have been used to determine the kinematics within their emission regions (Zickgraf 2003; Kraus et al. 2007, 2010, 2016; Maravelias et al. 2018; Torres et al. 2018).

The spectral energy distribution of $\mathrm{B}[\mathrm{e}] \mathrm{SGs}$ displays strong infrared (IR) excess emission due to hot and warm circumstellar dust (Allen \& Glass 1976; Zickgraf et al. 1986; Bonanos et al. 2009, 2010), and their IR colors have been proven suitable to distinguish B[e]SGs from other types of massive stars, such as Luminous Blue Variables and massive pre-main sequence objects (Oksala et al. 2013; Kraus 2019; Cochetti et al. 2020). Furthermore, the IR spectra of B[e]SGs provide complementary information about their environments. Most valuable in this regard is the molecular emission of $\mathrm{CO}$ (McGregor et al. 1988; Morris et al. 1996; Cidale et al. 2012; Oksala et al. 2012; Wheelwright et al. 2012; Kraus et al. 2014) and its isotope ${ }^{13} \mathrm{CO}$ (Liermann et al. 2010; Oksala et al. 2013; Kraus et al. 2020) from the circumstellar disks. The detection of an enrichment in the isotope is clear evidence that the disks have been formed from stellar wind material that has been enriched with chemically processed matter mixed to the stellar surface during the evolution of the star (Kraus 2009).

Emission from $\mathrm{SiO}$ has also been detected in a sample of B[e]SGs (Kraus et al. 2015). The kinematics of the molecular gas, obtained from high-resolution spectra, provides further information on the global disk dynamics. Under the assumption of strict Keplerian rotation, the combined velocity information from the optical forbidden lines and the molecular emission suggests that $\mathrm{B}[\mathrm{e}]$ SGs are surrounded by either multiple ring structures or disks with strongly varying density in radial direction (Maravelias et al. 2018). Moreover, the rings around some objects seem to display inhomogeneities as is inferred from the temporal variability of the emission features (Kraus et al. 2016; Torres et al. 2018).

The Galactic object MWC 137 (V1308 Ori, $\alpha=$ $06: 18: 45.52, \quad \delta=+15: 16: 52.24$, J2000) is a peculiar emission-line star, surrounded by the optical nebula Sh 2-266. Its evolutionary state was long unclear. Its resemblance with massive pre-main sequence stars led to the inclusion of the object into catalogs of Herbig Be stars (e.g., Hillenbrand et al. 1992; Berrilli et al. 1992; The et al. 1994), whereas the large scale environment with an optical nebula (Marston \& McCollum 2008), embedded in a dusty and cold molecular gas component (Kraus et al. 2017) which are reminiscent of the bipolar lobes and equatorial rings seen in the B-type supergiants Sher 25 and SBW 1 (Brandner et al. 1997; Smith et al. 2007), questioned the young nature.
Emission from the isotope ${ }^{13} \mathrm{CO}$ has been detected from MWC 137 in medium-resolution K-band spectra (Oksala et al. 2013; Liermann et al. 2014), and a ratio of ${ }^{12} \mathrm{CO} /{ }^{13} \mathrm{CO}=25 \pm 2$ has been determined by Muratore et al. (2015) from their high-resolution data. This enrichment in ${ }^{13} \mathrm{CO}$ is an unmistakable sign for chemically processed matter around the central object (Kraus 2009) and hence excludes a pre-main sequence nature of MWC 137. An evolved, supergiant classification of MWC 137 was also postulated by Esteban \& Fernandez (1998) due to the high stellar luminosity, and is reinforced by Mehner et al. (2016) based on the position of the star in optical and near-infrared color-magnitude and color-color diagrams. But despite the clear exclusion of a pre-main sequence nature, MWC 137 continues to appear in catalogs and studies about Herbig Be stars (e.g., Ababakr et al. 2017; Vioque et al. 2018; Arun et al. 2019), possibly owing to the star's peculiar characteristics.

A compact gaseous disk with a position angle (PA) of $120^{\circ}$ on the sky is inferred from $\mathrm{H} \alpha$ spectro-polarimetric observations (Oudmaijer \& Drew 1999; Vink et al. 2002). While the near- and mid-infrared colors point towards warm dust around MWC 137 (Mehner et al. 2016; Kraus 2019), there is a clear lack of cool dust in the close vicinity of the star (Sandell et al. 2011). Moreover, the spectral energy distribution at millimeter and centimeter wavelengths is dominated by free-free emission (Fuente et al. 2003).

An optical high-velocity jet with several knots has been discovered as well (Mehner et al. 2016), that seems to emanate from MWC 137 . With a PA of $18-20^{\circ}$, the jet is perpendicular to the postulated disk, but the origin of both jet and disk is still unclear.

High-resolution K-band spectra of MWC 137 show molecular emission from $\mathrm{CO}$ with a kinematic broadening of $84 \pm 2 \mathrm{~km} \mathrm{~s}^{-1}$ projected to the line of sight (Muratore et al. 2015), and the emission has been interpreted as coming from a molecular gas ring, possibly revolving the star on a Keplerian orbit. Additional indication for rotating circumstellar gas is provided by the intense optical forbidden emission lines of [O I] $\lambda \lambda$ 6300, 6364 . Maravelias et al. (2018) interpret the double-peaked profiles with broad wings of the [O I] lines with a superposition of four distinct ring contributions having rotation velocities, projected to the line of sight, of $68.0,46.8$, 31.0 , and $20.3 \mathrm{~km} \mathrm{~s}^{-1}$. Neither emission from [O I] $\lambda 5577$ nor from [CaII] has been detected from MWC 137 that could help to further constrain the disk structure and its kinematics.

A highly debated issue is the distance to MWC 137. Former estimates spread from $1.3 \mathrm{kpc}$ (Hillenbrand et al. 
1992) up to $13 \mathrm{kpc}$ (Fich \& Blitz 1984; Wouterloot et al. 1988). Based on the parallax measurement of $0.109624 \pm 0.054560$ mas provided by Gaia DR2, Arellano-Córdova et al. (2020) derived a distance of $13.6_{-1.3}^{+1.8} \mathrm{kpc}$ whereas Vioque et al. (2018) reported a value of $2.9074_{-0.4011}^{+0.6048} \mathrm{kpc}$ using the same parallax. The Gaia Early Data Release 3 (Gaia Collaboration et al. 2016, 2021) provides a refined value of $0.19404 \pm 0.02561$ mas for the parallax, which is about $77 \%$ higher than the previous one. Given the uncertainties in determining distances from measured parallaxes, we use the simple conversion with $d=1 / \mu$, where $\mu$ is the measured parallax, and obtain a value of $5.15_{-0.60}^{+0.79} \mathrm{kpc}$. While we are aware that this conversion bears uncertainties (see Luri et al. 2018), this value agrees reasonably well with the distance of $5.2 \pm 1.4 \mathrm{kpc}$ derived by Mehner et al. (2016) from an analysis of the cluster stars within Sh 2-266. Therefore, we will adopt a distance of $5.15 \mathrm{kpc}$ to MWC 137 .

In an attempt to determine the large-scale nebular kinematics Kraus et al. (2017) had obtained three longslit spectroscopic observations covering $\mathrm{H} \alpha$ as well as the nebular lines of $[\mathrm{N} \mathrm{II}]$ and $[\mathrm{S} \mathrm{II}]$. Their analysis of the emission lines revealed a complex behavior of the radial velocities across the nebula. However the number of slit positions was insufficient to derive the global kinematics.

In this paper, we present new data sets that have been acquired to complement the previous observations with the aim to achieve a more complete prescription of the nebular kinematics. Our various data sets and their reduction procedures are introduced in Section 2. In Section 3 we present our analyses of the nebula expansion and kinematics, along with the computation of the electron densities across the nebula. In this section we also investigate the photometric light curve of the central object. Our discussion on the nebula, stellar variability, and stellar parameters follow in Section 4, before we summarize our results in Section 5 .

\section{OBSERVATIONS AND DATA REDUCTION}

\subsection{Optical long-slit spectroscopy}

Four long-slit optical spectra were obtained on 2018 November 8 with the Nordic Optical Telescope (NOT) using the Andalucia Faint Object Spectrograph and Camera (ALFOSC). ALFOSC's field of view (FOV) is $6.4 \times 6{ }^{\prime} 4$ and the pixel scale is $0^{\prime \prime} 21 \mathrm{pix}^{-1}$.

We utilized Grism \#17 with a slit width of 0.5 , covering a spectral range of $6315-6760 \AA$ with a spectral reciprocal dispersion of $0.29 \AA \mathrm{pix}^{-1}(R \sim 10000)$. This choice is the same as for our previous observations (Kraus et al. 2017), covering $\mathrm{H} \alpha$ and the nebular lines [N II] $\lambda \lambda 6548,6583$ and [S II] $\lambda \lambda 6716,6731$.

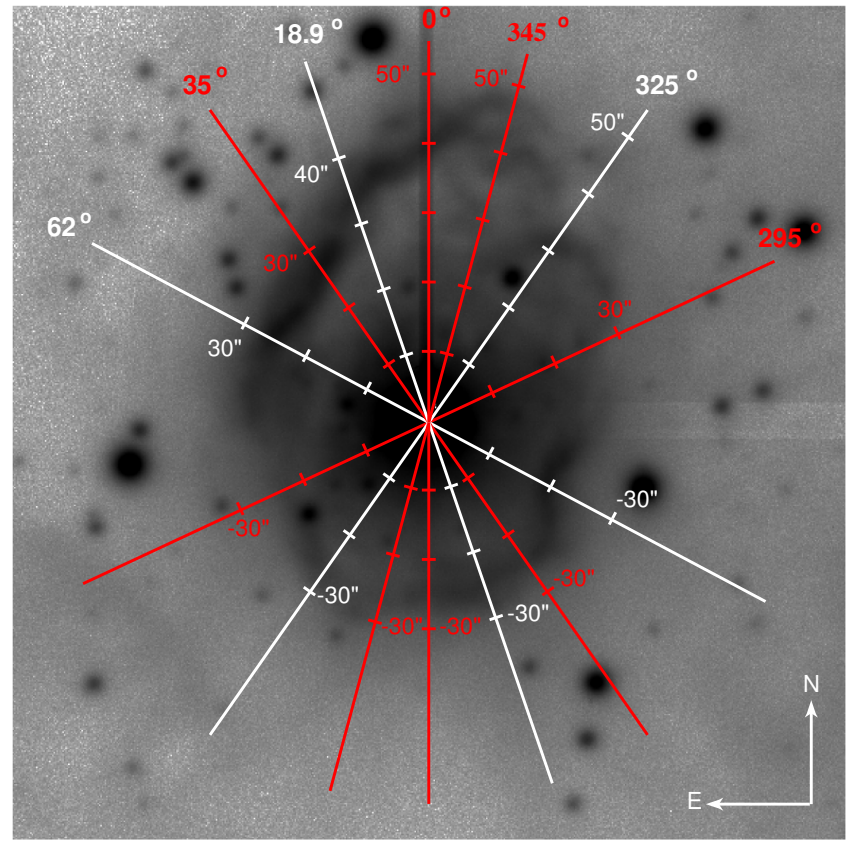

Figure 1. ALFOSC $\mathrm{H} \alpha$ image of the nebular structure around MWC 137 from our previous observations in 2016 (Kraus et al. 2017). The FOV is $2^{\prime} \times 2^{\prime}$. The new (red) slit positions have been included along with the old (white) ones. PAs are marked with boldface numbers. The distance scale in steps of $10^{\prime \prime}$ is marked with ticks along the slits in both directions from the central star.

The slits were centered on the star and had PAs of $0^{\circ}$, $35^{\circ}, 295^{\circ}$, and $345^{\circ}$ (see Figure 1 ) following the convention of measuring PAs from North to East ${ }^{1}$. For each slit position the exposure time was $30 \mathrm{~min}$, and the observations were carried out with a seeing of $\sim 1^{\prime \prime}$.

The spectra were reduced (bias, flat fielding, wavelength calibration) using standard $\mathrm{IRAF}^{2}$ routines, and were corrected for both the heliocentric and the systemic radial velocity (RV). For the latter we used the value of $42 \mathrm{~km} \mathrm{~s}^{-1}$ determined by Kraus et al. (2017).

\subsection{Optical Fabry-Perot Interferometry}

Additional observations have been taken on 2019 November 19 and 21 with the Spectral Camera with Optical Reducer for Photometric and Interferometric Observations No 2 (SCORPIO-2, Afanasiev \& Moiseev 2011), mounted at the Russian 6-m telescope (BTA). We used SCORPIO-2 in the Scanning Fabry-Perot mode in

\footnotetext{
${ }^{1}$ Note that in Kraus et al. (2017) the PAs were accidentally measured from North to West.

${ }^{2}$ IRAF is distributed by the National Optical Astronomy Observatory, which is operated by the Association of Universities for Research in Astronomy (AURA) under cooperative agreement with the National Science Foundation.
} 
the order 751, operating with 40 spectral channels and providing a spectral resolution of $0.4 \AA(R \sim 15400)$. The total field of view of SCORPIO- 2 is $6.1 \times 6.1$. The CCD detector, E2V 42-90, has a size of $4600 \times 2048$ pixels with a pixel size of $13.5 \times 13.5 \mu \mathrm{m}$ and a pixel scale of 0 .'18 per pixel. During the observations with the FPI only the central part of the detector was used in $4 \times 4$ read-out binning mode providing the scale 0.71 per pixel.

The observations were centered on the [S II $] \lambda \lambda$ 6716,6731 lines. We refrained from using the filter covering $\mathrm{H} \alpha$ and the adjacent [N II] $\lambda 6583$ line because of the brightness of the central star in $\mathrm{H} \alpha$ leading to strong saturation and blending effects when scanning the central regions. Instead, we gave preference to the two nebular lines [S II]. Their lower intensities allowed us to take longer exposures, hence deeper images, without the risk of saturation.

During the first observing night, the observations were obtained in a single orientation, whereas two different orientations were used during the second night. The different orientations help to correct for parasitic ghosts. For each set of observations, the exposure times were $30 \mathrm{sec}$ per channel. Data reduction was performed following the recipes of Moiseev (2002, 2015) and Moiseev \& Egorov (2008). The reduced data cubes for the two orientations from the second night have been co-added. Unfortunately, the observing conditions were poor, with seeing changing from 2 . $7-3$.' 5 to 3 .' $1-3$.' 8 between the two observations. To combine the two data cubes, we had to degrade the one taken with the slightly better conditions, resulting in a final spatial resolution of just 3 "' 8 . The data cube from the first night was taken under significantly better seeing conditions of $1 . ! 2-1 . ! 5$, resulting in a final spatial resolution of $1^{\prime \prime} .5$.

The data have been corrected for heliocentric and systemic RV and continuum subtracted. Moreover, for each [S II] line the data have been converted into RV, and the emission in both lines has been co-added in velocity space for a better signal to noise ratio (SNR) of the nebular structure. The final SNR has values spreading from 5-10 in the faintest to 55-60 in the brightest nebular regions.

\subsection{Optical imaging data}

A new $\mathrm{H} \alpha$ image of the MWC 137 nebula was acquired with the 1.54-m Danish Telescope at La Silla on 2019 October 27. The Danish Faint Object Spectrograph and Camera (DFOSC) was exploited with a FOV of $13.7 \times$ $13 ! 7$ and a pixel scale of $0.39 \mathrm{pix}^{-1}$. The narrow band $\mathrm{H} \alpha$ filter, ESO \#693, centered at $656.23 \mathrm{~nm}$ and with a width of $6.21 \mathrm{~nm}$, was used. The total exposure time was $35 \mathrm{~min}$, achieved with 7 dithered single exposures of 5 min each. The seeing of the combined frame was $1^{\prime \prime} 5$.

In addition, we have at our disposal the raw images of MWC 137 presented by Marston \& McCollum (2008). A single, 20 min long exposure was acquired on 2001 September 28 with the 60 -inch telescope on Mt. Palomar with a seeing of 2 . 1 . The used FOV, pixel scale and narrow band $\mathrm{H} \alpha$ filter are all comparable to our observations with the Danish Telescope. For more details on these observations see Marston \& McCollum (2008).

For both facilities, standard routines in IRAF were used for data reduction and for combining the individual frames.

\subsection{Complementary data}

To date, MWC 137 (TIC 437994564) has been observed twice with high-cadence $(120 \mathrm{~s})$ by the Transiting Exoplanet Survey Satellite (TESS). The first observation period was between 2018 December 15 and 2019 January 6 (BJD $=2458468.27-2458490.05)$ in Sector 6 with camera 1 and CCD 3 . The second visit took place about two years later, between 2020 December 18 and 2021 January 13 (BJD = 2459201.74-2459227.57) in Sector 33 with camera 1 and CCD 4 . We accessed the calibrated light curves ${ }^{3}$ through the Mikulski Archive for Space Telescopes (MAST). Calibrated means that the flux series has been corrected for common instrumental systematics, for the amount captured by the photometric aperture, and for crowding from known nearby stars. From these calibrated TESS light curves we used only the highest quality measurements for the analysis.

\section{RESULTS}

\subsection{Possible expansion in the plane of the sky}

Our new image of MWC 137 from 2019 was used in comparison to the image from 2001 to explore any morphological changes and/or expansion of the large scale nebula in the plane of the sky over the total time span of 18.1 years. We utilized more than 40 stars in the FOV to first match both images, pixel by pixel, using the IRAF tasks geomap and geotran. The RMS of the matching was 0.1 pixels. From a first glance, no obvious morphological evolution or expansion of the nebula was seen from a simple by-eye inspection (blinking) of the images.

Next, we used the so-called magnification method (see e.g. Reed et al. 1999; Liimets et al. 2018) which uses the residual images to detect displacements in the nebula

\footnotetext{
3 The data described here may be obtained from the MAST archive at https://dx.doi.org/10.17909/t9-ncv5-bb52.
} 

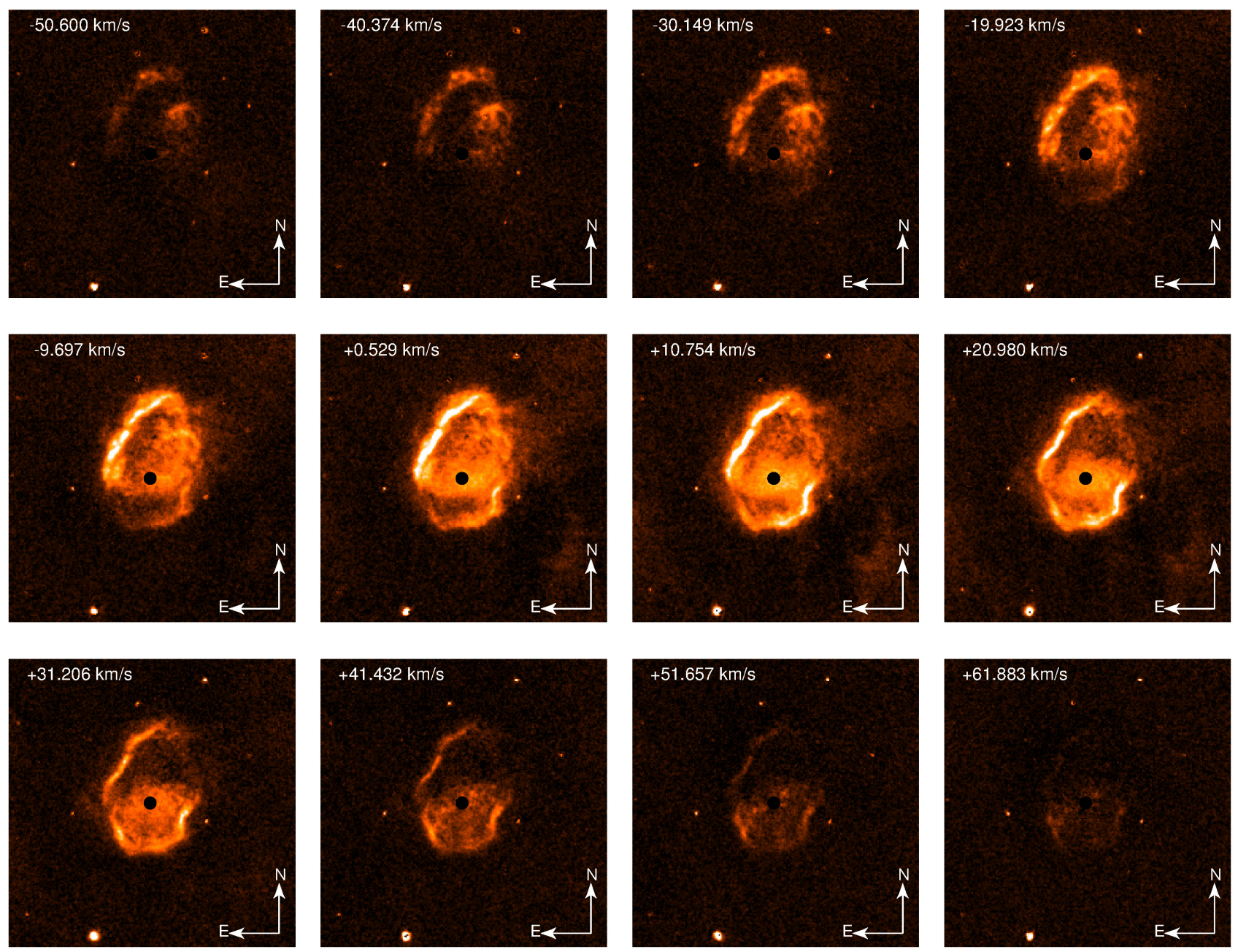

Figure 2. FPI images of the co-added $[\mathrm{S} \mathrm{II}] \lambda \lambda 6716,6731$ lines in various velocity bins. The image sizes are $3^{\prime} \times 3^{\prime}$ and have been centered on the stellar position. The central regions with a radius of $2.5^{\prime \prime}$ have been masked. The intensity scale is linear and is the same in all images.

down to 0.1 pixels. For this, further image processing was required, such as matching the seeing of the 2019 image to the slightly worse one of the 2001 image, and matching the flux. This matching was performed as described in Section 3 and 4.1 in Liimets et al. (2018).

After that, the 2001 frame was magnified, using the IRAF task geotran with a steadily increasing magnification factor, and then subtracted from the 2019 image. If the nebula would have expanded in the plane of the sky, the resulting residual images should reveal a distinct pattern based on which the magnification could be determined (see Reed et al. 1999). However, our analysis revealed the we detected neither any morphological changes nor an expansion in the plane of the sky.

This means that, considering the time span of 18.1 years, a possible expansion in the plane of the sky is proceeding with a tangential velocity of less than $52.7 \mathrm{~km} \mathrm{~s}^{-1}$ for the pixel scale of $00^{\prime \prime} 39$ and the distance of $5.15 \mathrm{kpc}$ towards MWC 137 . In fact, the maximum
RV component measured in the nebula is only about $40 \mathrm{~km} \mathrm{~s}^{-1}$ (see Kraus et al. 2017, and Section 3.3 below) and hence too small to create detectable changes in the nebular morphology to date. For the idealized case of a ballistically expanding sphere with a tangential velocity of $40 \mathrm{~km} \mathrm{~s}^{-1}$, we can hence expect to detect an indication of expansion earliest after a time span of about 24 years, that is in the year 2025. However, the lack of significant RV of the nebula around the position of the star questions a ballistic expansion so that the real expansion of the nebula in the plane of the sky might proceed (much) slower.

\subsection{The FPI images}

Although the data from the second night had longer exposure times, we present the data from the first night because of their significantly higher spatial resolution. The lack of a second orientation observation resulted in several artefacts that appear on the final cube, such 


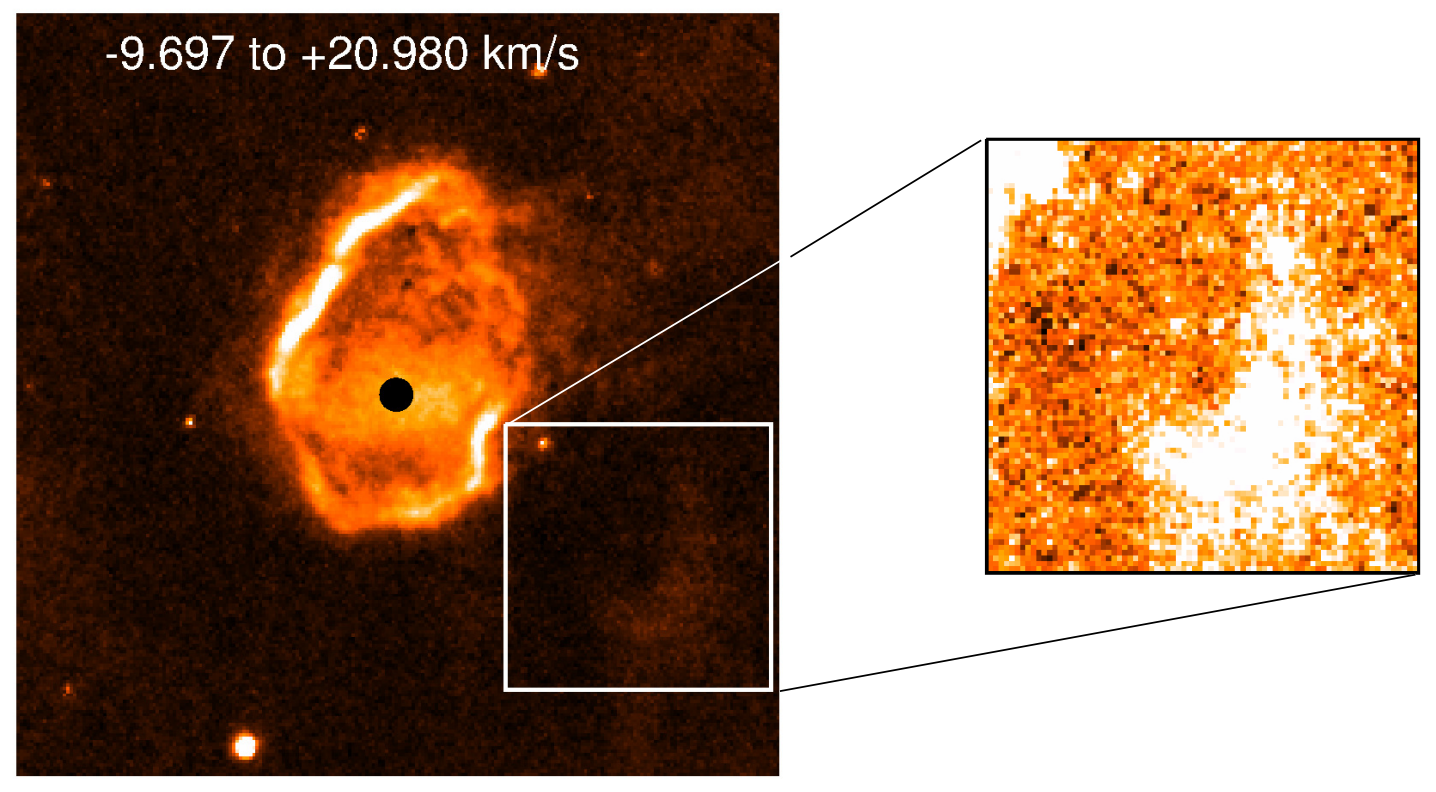

Figure 3. Co-added [S II] emission in the velocity range $-10 \mathrm{~km} \mathrm{~s}^{-1}$ to $+21 \mathrm{~km} \mathrm{~s}^{-1}$. North is up and East is to the left. Ionized gas seems to emanate from the north-western nebular regime causing diffuse emission out to large distances, and a bow structure is detected in the south-western region. This bow is marked with the white box and magnified in the right panel using a higher intensity contrast.

as false emission at both the stellar position and the position of several stars in the vicinity. These could not be properly corrected but were identified based on the deeper data cube. Within the nebula structure, only at the central star's position a ghost artifact was identified and masked and is excluded from our discussion. This masked area around MWC 137 has a radius of 2.5", corresponding to a sphere with a radius of $0.062 \mathrm{pc}$.

Slicing the FPI data cube with respect to RV, we find that the nebula is detected from about $-51 \mathrm{~km} \mathrm{~s}^{-1}$ to about $+62 \mathrm{~km} \mathrm{~s}^{-1}$ with respect to the nebular systemic velocity, as shown in Figure 2. For a better comparison, the intensity in all velocity bins is displayed on a linear scale, and in all panels the intensity range is the same.

Starting from the very blue, the first features that become visible are two structures in the northern nebular regions. The north-eastern ring or arm-like structure brightens until it reaches maximum intensity at $+0.5 \mathrm{~km} \mathrm{~s}^{-1}$ and then starts to fade and disappear for velocities redder than $+52 \mathrm{~km} \mathrm{~s}^{-1}$. The second, most blue-shifted emission is related to a smaller ring or armlike structure north-west of MWC 137. The northern domain of this feature reaches maximum intensity at $-20 \mathrm{~km} \mathrm{~s}^{-1}$ and disappears for velocities redder than $+11 \mathrm{~km} \mathrm{~s}^{-1}$. Starting from about $-30 \mathrm{~km} \mathrm{~s}^{-1}$, a southwestern arm starts to appear. Its maximum intensity is reached around $+11 \mathrm{~km} \mathrm{~s}^{-1}$, and it remains visible beyond red-shifted velocities of $+52 \mathrm{~km} \mathrm{~s}^{-1}$. Around $+11 \mathrm{~km} \mathrm{~s}^{-1}$, this feature seems to connect to the large north-eastern arm structure. A further, high intensity region appears west of the central object in the velocity interval $-10 \mathrm{~km} \mathrm{~s}^{-1}$ to $+20 \mathrm{~km} \mathrm{~s}^{-1}$. In the velocity bins at $+0.5 \mathrm{~km} \mathrm{~s}^{-1}$ and $+11 \mathrm{~km} \mathrm{~s}^{-1}$, this emission seems to spread around the central object in east-west direction. Finally, the south-western arm-like structure embraces some diffuse emission in the southern region with velocities from $+31 \mathrm{~km} \mathrm{~s}^{-1}$ to approximately $+52 \mathrm{~km} \mathrm{~s}^{-1}$.

Furthermore, the large FOV of the FPI reveals emission features at greater distances from the star, in regions which have not been covered by the MUSE mosaic of Mehner et al. (2016). These are (i) diffuse emission apparently emanating from the north-western nebular regime, and (ii) a bow structure towards the south-west at a PA of $225-245^{\circ}$. Both emission structures are visible on our $\mathrm{H} \alpha$ images taken with ALFOSC and with the Danish telescope, and are also present on the image of Marston \& McCollum (2008). But Marston \& McCollum (2008) mentioned only the diffuse emission. The presence of the bow structure on all images taken between 2001 and 2019 and with different instruments implies that it is real and not an artifact.

These two emission features appear in the velocity interval from $-10 \mathrm{~km} \mathrm{~s}^{-1}$ to $+21 \mathrm{~km} \mathrm{~s}^{-1}$. For better visibility of these features, we co-added the images in this velocity range and show the combined image in Figure 3. The vertex of this bow or arch has a distance of about $80^{\prime \prime}$ from the central star and an extension of about $36^{\prime \prime}$ from north-west to south-east. These measurements 

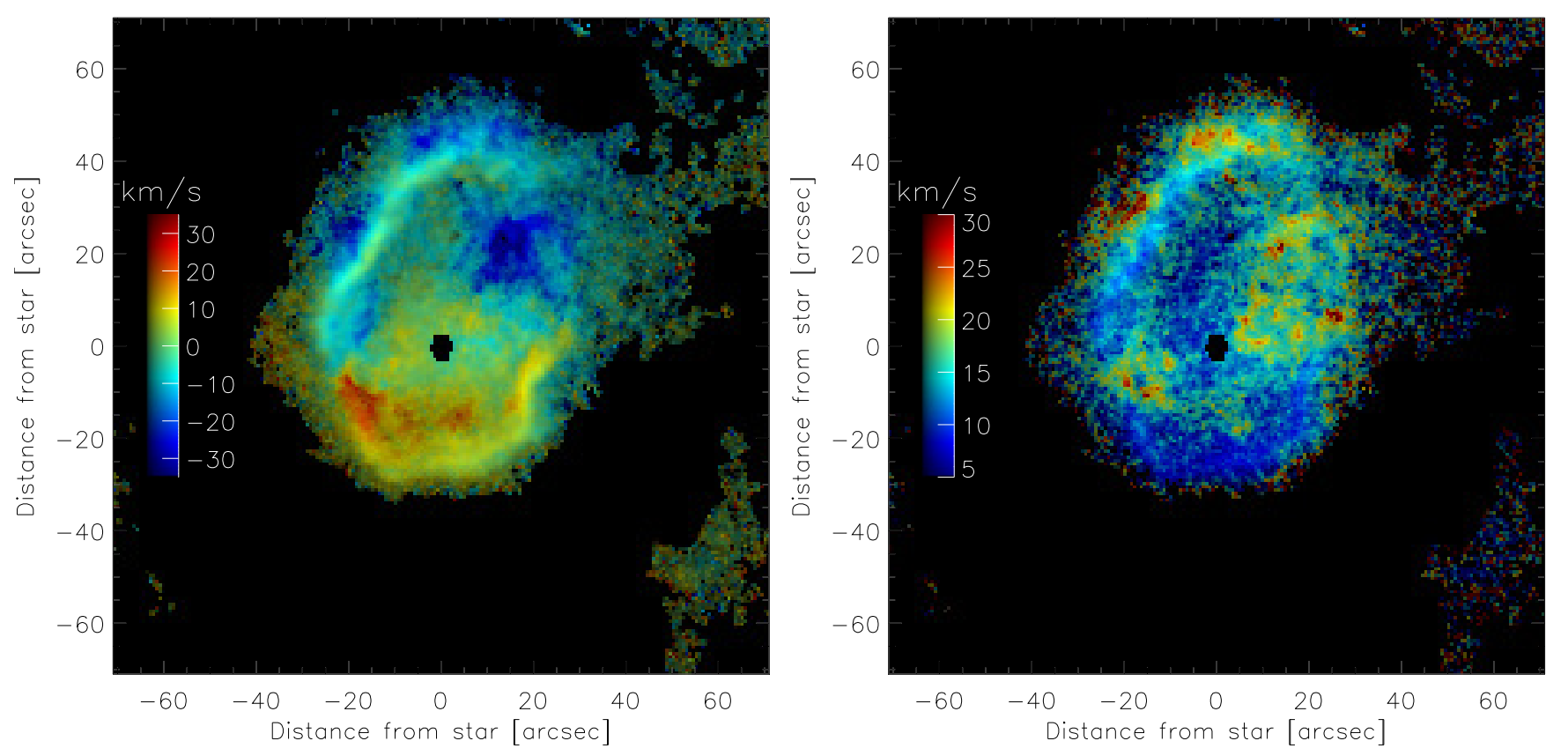

Figure 4. FPI images of the $[\mathrm{SII}]$ emission. North is up and East is to the left. In color are shown the radial velocity distribution (left panel) and the velocity dispersion (right panel) corrected for instrumental broadening. The pixel brightness is scaled according to the [S II] line intensity.

translate into physical scales, projected to the plane of the sky, of $2 \mathrm{pc}$ and $0.9 \mathrm{pc}$. But whether its origin is linked to MWC 137 is difficult to tell. At least, this arch or bow seems not to be related with the jet discovered by Mehner et al. (2016), which emerges at a PA of $18-20^{\circ}$.

Finally, we use the FPI data cube to measure the radial velocities and velocity dispersions of the combined [S II] lines over the full nebula. Visual inspection of the profiles reveals that the lines are single-peaked everywhere in the nebula. The emission lines are fitted with a Voigt profile, and the velocity dispersion has been corrected for instrumental broadening as described in Moiseev \& Egorov (2008). The results are shown in Figure 4. The general trend is that the intense nebula arm structures have lower red- or blue-shifted velocity compared to the gas inside and outside (in projection onto the plane of the sky) of these structures. In addition, we observe that the regions with highest radial velocities have the tendency to also display high velocity dispersion, but with no strict coincidence.

The choice of the filter setting for these observations was optimized such that both [S II] lines were simultaneously observed. However, as we can see in Figure 4, the emission can become rather broad in some nebular regions, so that not always the full profile of either the blue or the red [S II] line was covered by the narrow filter, hampering a more detailed analysis of the physical conditions across the entire nebula.

\subsection{Radial velocities along the long-slit spectra}

The long-slit spectra taken with ALFOSC cover, besides $\mathrm{H} \alpha$, the forbidden emission lines [N II] $\lambda \lambda$ 6548,6583 and $[\mathrm{SII}] \lambda \lambda 6716,6731$. The slightly better observing conditions under which these spectra were taken, compared to the FPI images and, in particular, the longer spectral coverage allow for more precise measurements of the kinematics, as well as a proper determination of the physical parameters of the nebula.

For both doublets we focused on their more intense line to perform the RV measurements. These are [N II] $\lambda 6583$ and, over large regions of the nebula, $[\mathrm{S} \mathrm{II}] \lambda$ 6716. For each line, we fit the emission profiles in the $2 \mathrm{D}$ spectra, line by line, to determine RV as a function of distance from the star. The lines appear single-peaked and are not blended so that we fit them with a single Gaussian profile. These measurements are presented for all four PAs in Figure 5 and are provided in an on-line table following the layout of Table 1. Profiles were excluded from the measurements if they were (i) too weak, (ii) contaminated by a cosmic ray that could not be removed, or (iii) contaminated with a neighboring line. The latter happens especially for the [N II] $\lambda 6583$ line in the vicinity of the central star where $\mathrm{H} \alpha$ is saturated, preventing any precise measurements of that line. The uncertainties of the velocity measurements are rather small, with an average value of $0.95 \mathrm{~km} \mathrm{~s}^{-1}$, and are included in Table 1. They have been determined from the 

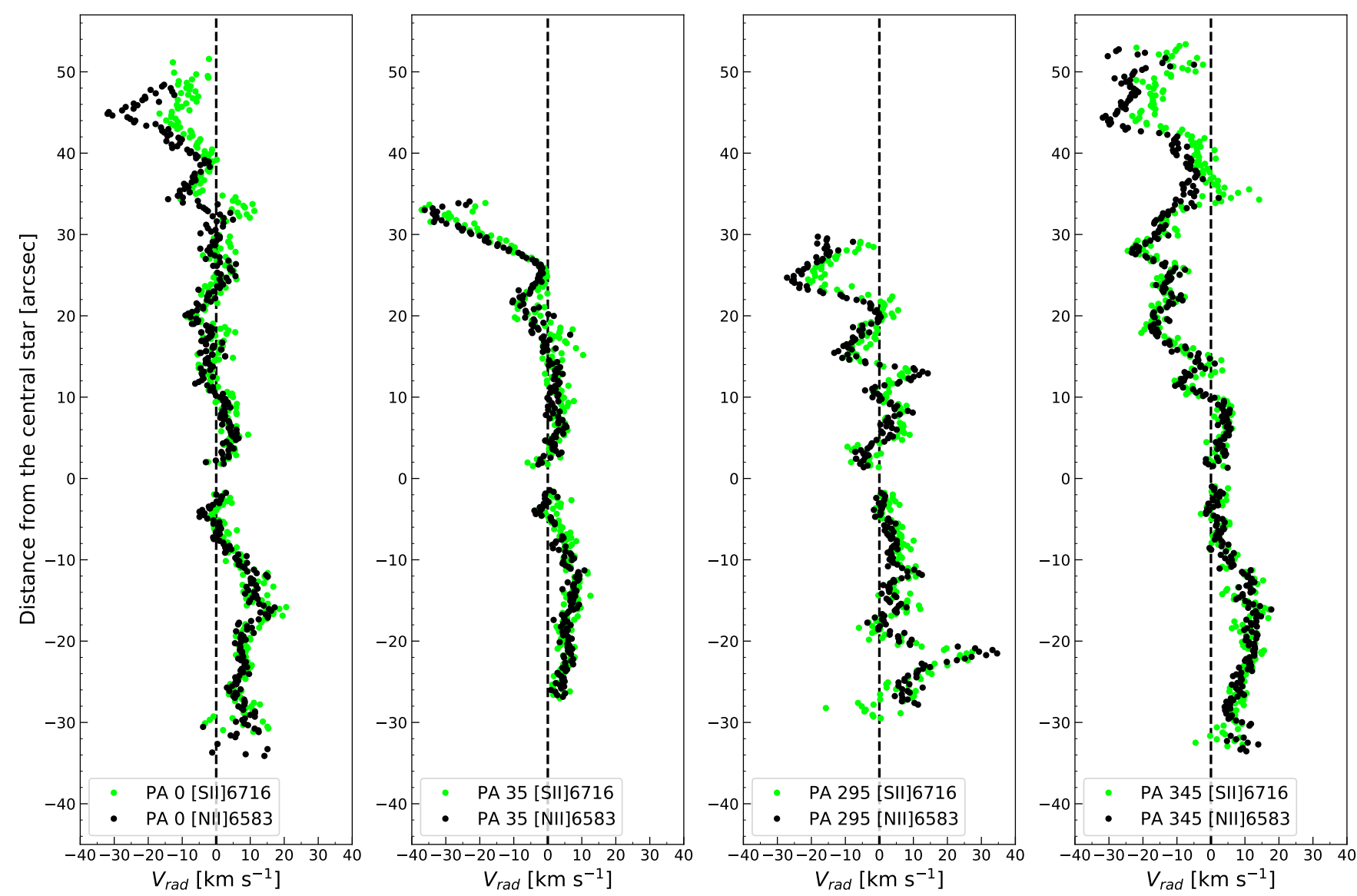

Figure 5. Radial velocities of the $[\mathrm{N} \mathrm{II}] 6583 \AA$ (black) and [S II] $6716 \AA$ (green) emission lines. Positive distance from the central star on the image is towards the North, negative towards the South.

RMS of the wavelength calibration. Contributions from the fitting to the total error are negligible.

From Figure 5 we can see that in general both nebular lines display the same trend in the gas dynamics. However, some deviations are noticeable. First, in the close vicinity of the hot central star the $[\mathrm{S} \mathrm{II}]$ lines disappear and the $[\mathrm{N} \mathrm{II}]$ lines become very weak on all our spectra. This points towards a higher electron density favoring collisional depopulation of the levels, along with a hotter gas and, therefore, preferentially higher ionized material. In fact, the higher density is obvious from the much more intense $\mathrm{H} \alpha$ emission towards the center, whereas the slightly higher ionization state follows from high-resolution spectra of the innermost $2^{\prime \prime}$ that display considerable emission of [S III] $\lambda 6312$ and only very weak emission in [S II] $\lambda 6716$ (Kraus et al. 2017, their Figure 3). These hindrances in measurements result in a gap in the RV values in all PAs within a radius of about $1 . .5-2$." 0 from the central star.

Second, we notice considerable differences in the RVs of the two lines in the regions with highest blue-shifted
Table 1. Radial Velocity Measurements

\begin{tabular}{lcrcc}
\hline \hline PA & $\begin{array}{c}\lambda_{\text {lab }} \\
\left({ }^{\circ}\right)\end{array}$ & $\begin{array}{c}\text { RV } \\
(\AA)\end{array}$ & $\begin{array}{c}\text { RV uncertainty } \\
\left(\mathrm{km} \mathrm{s}^{-1}\right)\end{array}$ & $\begin{array}{c}\text { Dist } \\
\left(\mathrm{km} \mathrm{s}^{-1}\right)\end{array}$ \\
\hline 0 & 6716.440 & 10.90 & 0.94 & -31.15 \\
0 & 6716.440 & 2.02 & 0.94 & -30.94 \\
0 & 6716.440 & 15.32 & 0.94 & -30.73 \\
0 & 6716.440 & 15.05 & 0.94 & -30.52 \\
0 & 6716.440 & 7.91 & 0.94 & -30.31 \\
\hline
\end{tabular}

Note-The entire table is published only in the electronic edition of the article. The first 5 lines are shown here for guidance regarding its form and content.

RV values towards to north and north-west for PAs at $0^{\circ}, 325^{\circ}$, and $295^{\circ}$ (the same is seen for PA $345^{\circ}$, Kraus et al. 2017) where the [N II] displays significantly higher velocities than the $[\mathrm{S} I \mathrm{I}]$. We see the same trend in the red-shifted RV maximum for $\mathrm{PA}=295^{\circ}$ in the south-east. The deviations can reach values of $\Delta v_{\text {rad }} \approx 10-15 \mathrm{~km} \mathrm{~s}^{-1}$. Because these differences are 


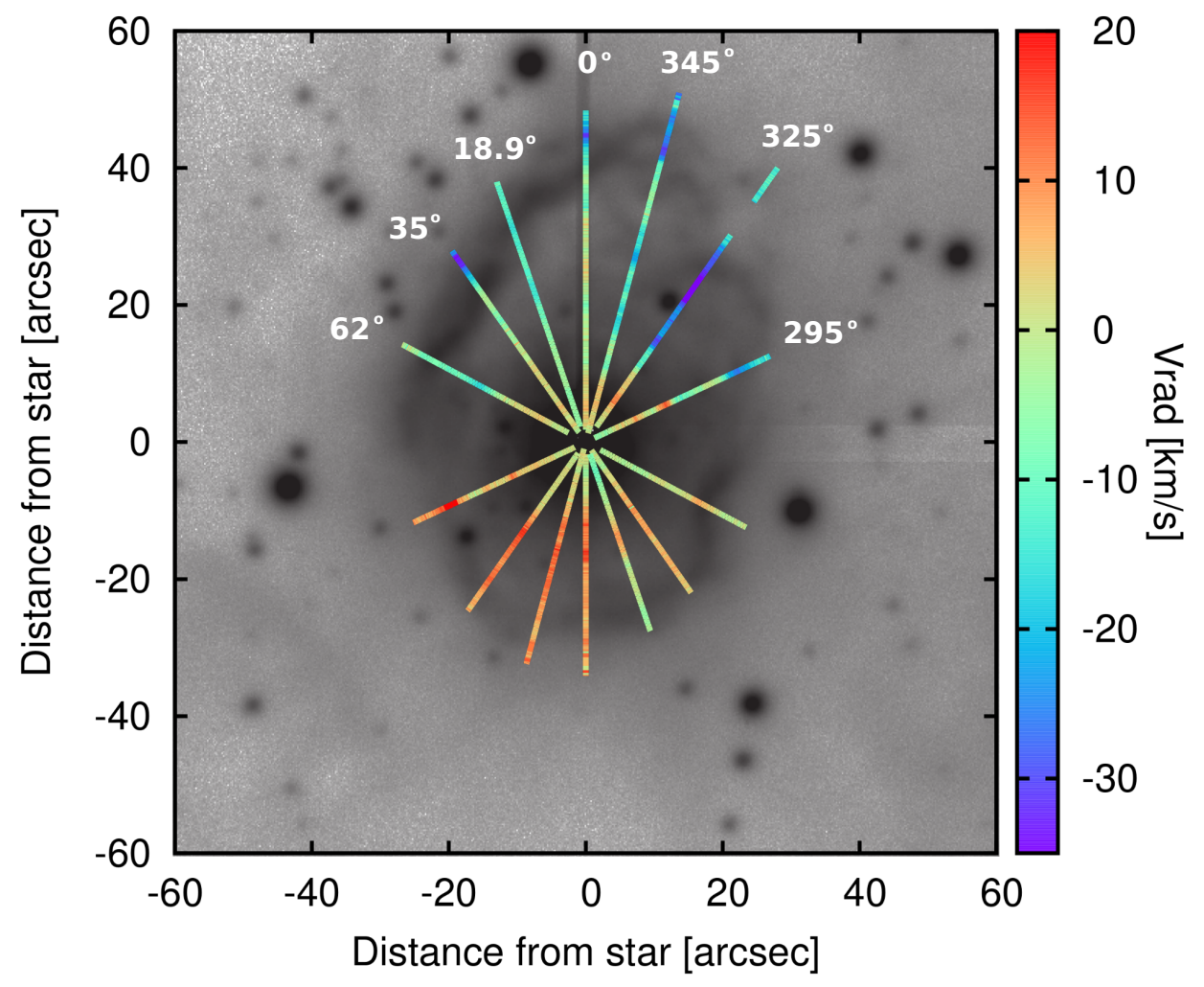

Figure 6. Color presentation of the radial velocities along the different slits measured from the [N II] $6583 \AA$ line.

significantly larger than the measurement errors, they are considered as real. Taking into account that the ionization potential of $\mathrm{N}$ I (I.P. $=14.5 \mathrm{eV})$ is higher than that of S I (I.P. $=10.4 \mathrm{eV})$, and that the ionization of the elements is governed by the radiation flux from the hot central star, the emission in [N II] should be formed closer to the star than the emission in [SII]. A relation between RV and the ionization potentials of the elements has been found in H II regions (see, e.g., Esteban \& Peimbert 1999; Caicedo-Ortiz \& Fernandez 2019) where it has been used to trace the velocity stratification along the line of sight. Application to the nebula of MWC 137 suggests that within these specific regions we see deceleration of the material from inside out.

In agreement with the FPI data, the long-slit spectra reveal that the northern nebular region is predominantly blue-shifted with respect to the nebular systemic velocity, reaching values up to $-36.2 \mathrm{~km} \mathrm{~s}^{-1}$, whereas the southern part is mostly red-shifted with velocities up to $+34.6 \mathrm{~km} \mathrm{~s}^{-1}$. But despite this general trend, we also note strong variability of the RV values along most PAs, with multiple maxima and minima, in agreement with the inhomogeneous velocity pattern across the nebula seen in Figure 4 and with the wiggly structures detected along the PAs presented by Kraus et al. (2017).
To visualize the RVs over the measured nebular regions, we utilize the RV values of the [N II] $\lambda 6583$ line and combine them with those of our previous long-slit spectra for the three PAs at $18.9^{\circ}, 62^{\circ}$, and $325^{\circ}$ (respectively at $341.1^{\circ}, 298^{\circ}$, and $35^{\circ}$ according to the notation in Figure 4 of Kraus et al. 2017). In Figure 6 we present the color-coded RVs along all seven slits. For a comparison with the nebular structures, these velocities are overlaid on the $\mathrm{H} \alpha$ emission nebula.

From this image we can deduce that the largest region displaying highly blue-shifted RVs with values exceeding $-40 \mathrm{~km} \mathrm{~s}^{-1}$ resides in the north-western area of the nebula, in particular along $\mathrm{PA}=325^{\circ}$. This area corresponds to the region in which neither cold molecular gas nor significant emission from warm dust is seen (Kraus et al. 2017). A further, though smaller blueshifted domain occurs towards the north-east, beyond the outer rim (maximum intensity in the $\mathrm{H} \alpha$ image) of the nebula at $\mathrm{PA}=35^{\circ}$. Here, the velocities reach values around $-36 \mathrm{~km} \mathrm{~s}^{-1}$ whereas they tend towards zero within the region of maximum intensity. A similar behavior is found for the most northern region along $\mathrm{PA}=$ $0^{\circ}$. Also here the RV is around zero on top of the maximum intensity and reaches highest blue-shifted values beyond that region. For the other PAs with blue-shifted emission $\left(295^{\circ}, 325^{\circ}\right.$, and $\left.345^{\circ}\right)$ the situation is differ- 

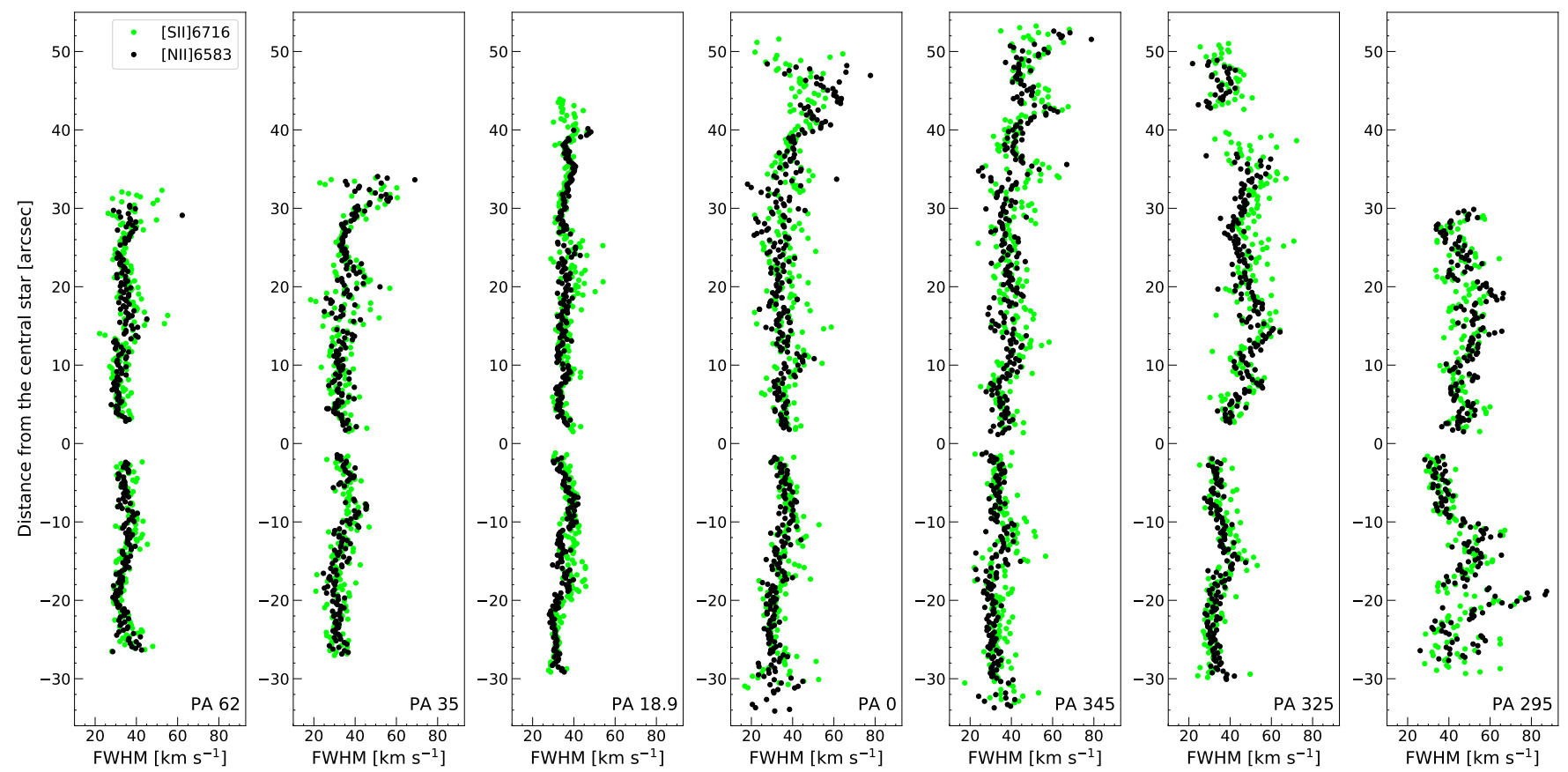

Figure 7. Measured FWHM values of the [N II] $6583 \AA$ (black) and [S II] $6716 \AA$ (green) emission lines along all seven PAs. Positive distance from the central star on the image is towards the North, negative towards the South.

ent. Here, the emission with maximum blue-shift either coincides with the intensity peaks or occurs at distances closer to the central star.

Turning to the southern nebular region we find the highest red-shifted velocities in the south-eastern portion, with a narrow peak of about $+35 \mathrm{~km} \mathrm{~s}^{-1}$ reached within an apparent gap in the $\mathrm{H} \alpha$ double-ring structure along PA $295^{\circ}$. For the PAs at $325^{\circ}, 345^{\circ}$, and $0^{\circ}$, the highest red-shifts occur (much) closer to the central star and are not correlated with the regions of maximum emission intensity. The only exception is $\mathrm{PA}=$ $62^{\circ}$. Here the red-shifted emission coincides with the maximum intensity. But the red-shift in that region is with values smaller than $+10 \mathrm{~km} \mathrm{~s}^{-1}$ much less prominent than along other PAs.

Our measurements also provide the FWHM values of the emission lines. These are shown in Figure 7 for $[\mathrm{N} \mathrm{II}]$ $6583 \AA$ and [S II] $6716 \AA$ along all seven PAs. As for the RV values, we find that both forbidden lines display the same behavior of their FWHM values, with noticeable differences only in regions in which the lines are very faint and thus have a higher measurement uncertainty. For most PAs, a significant portion of the nebula displays some average FWHM value of $30-40 \mathrm{~km} \mathrm{~s}^{-1}$ (whereby $30 \mathrm{~km} \mathrm{~s}^{-1}$ correspond to the velocity resolution of the long-slit spectra), but in certain regions it can reach values of $70-80 \mathrm{~km} \mathrm{~s}^{-1}$. Exceptions are the PAs at $325^{\circ}$ and $295^{\circ}$, which exhibit considerably higher
FWHM values over large nebular portions. We also find that the nebular areas with high FWHM values correlate more or less with the regions of high RV, in agreement with what is seen in Figure 4.

\subsection{Electron densities}

From their low-resolution MUSE data Mehner et al. (2016) estimated average values of $T_{e} \sim 10000 \mathrm{~K}$ and $n_{e} \sim 300 \mathrm{~cm}^{-3}$ for the electron temperature and density across the nebula. Our spectra have three times higher resolution, but are short in wavelength coverage and thus contain just two strategic forbidden lines, [S II] $\lambda \lambda 6716,6731$, which allow us to investigate the electron density distribution along the PAs, but we cannot estimate the electron temperature.

From our spectra, we measure, line by line, the line fluxes of both [S II] lines and then average the values every three lines before computing their ratio. This binning reduces the measurement errors, particularly in the very faint nebular regions, and also represents better the real values considering a seeing of $\sim 1^{\prime \prime}$ during the observations compared to the pixel size of 0.221 . A conservative uncertainty estimate of the flux measurements is $5 \%$ resulting in an uncertainty on the observed line ratios of about $7 \%$.

From theory, the intensity ratio of these two [S II] lines is given by

$$
\frac{I(6716)}{I(6731)}=\frac{N(6716) A(6716) \lambda(6731)}{N(6731) A(6731) \lambda(6716)}
$$



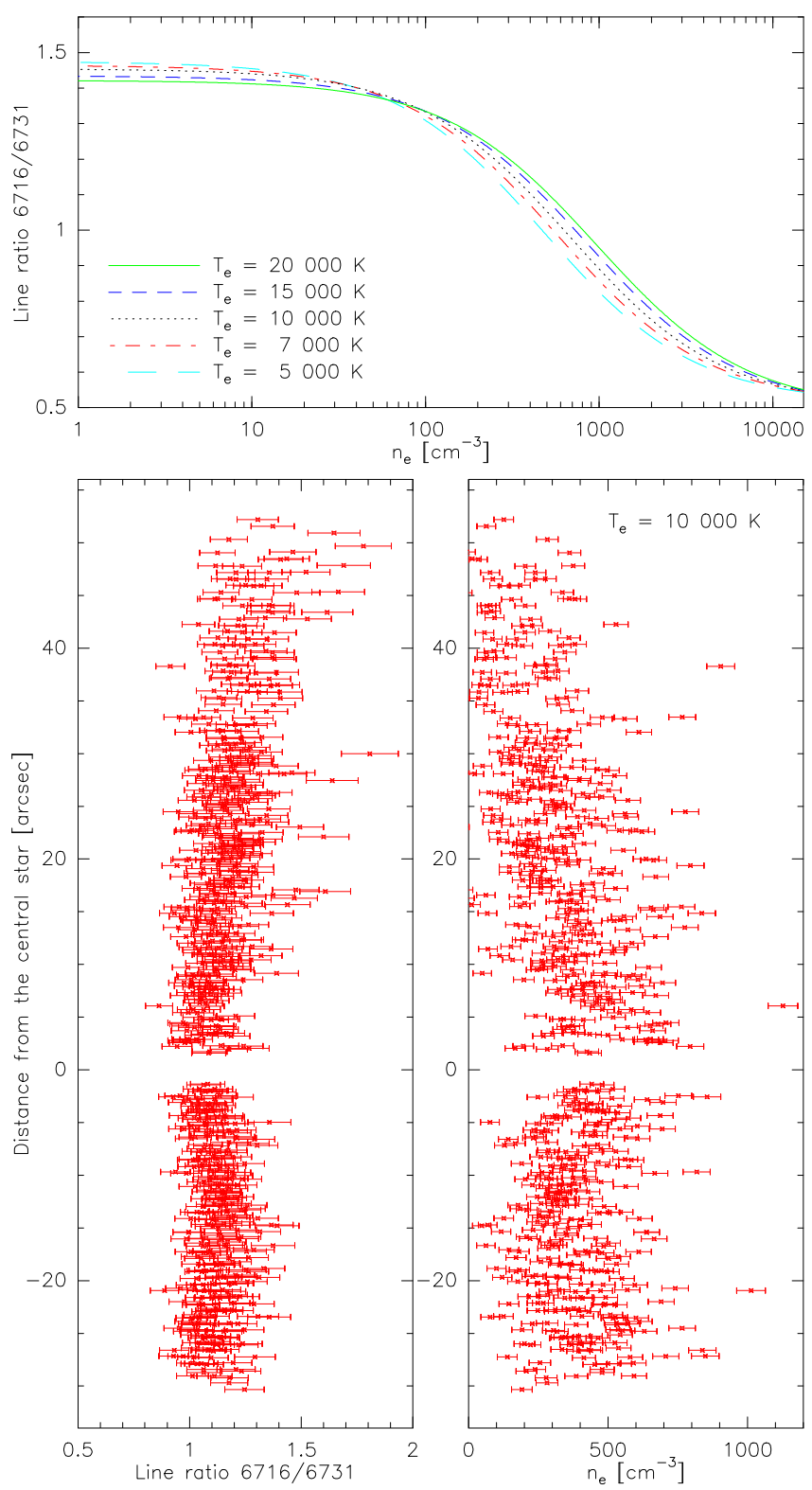

Figure 8. Top: theoretical line ratios as function of electron density and for different electron temperatures. Bottom: Observed line ratios (left) for the measurements along all PAs, and derived electron densities (right) for a nebular temperature of $10000 \mathrm{~K}$.

where the parameters $N, A$, and $\lambda$ denote the level population, Einstein transition probability, and laboratory wavelength of the corresponding transition. This ratio is an implicit function of the electron density for a given temperature and vice versa.

To compute the level population we consider a fivelevel atom in which the levels, from which the forbidden lines arise, are excited by collisions with free electrons. Collision parameters and Einstein transition probabilities are taken from Tayal \& Zatsarinny (2010). The level population is calculated for electron temperatures between $5000 \mathrm{~K}$ and $20000 \mathrm{~K}$ and for electron densities ranging from 1 to $15000 \mathrm{~cm}^{-3}$. The line ratios as a function of electron density are shown in the top panel of Figure 8 for five different electron temperature values, whereas the observed line ratios from North to South across the nebula are plotted in the bottom left panel, and the resulting electron densities, considering a constant electron temperature of the nebula of $10000 \mathrm{~K}$, in the bottom right panel.

The densities display high local fluctuations with the majority of measurements spreading from 200 to $500 \mathrm{~cm}^{-3}$. But we also find a clear increase in electron density towards the position of the central star. This can be seen in the color-coded image shown in Figure 9, where the electron density distribution along each PA is displayed and overlaid on the $\mathrm{H} \alpha$ emission nebula. From this image it becomes also evident that higher densities occur in the regions of high intensities. Moreover, in the north-western region where the RVs are highest, the density rapidly decreases. The gaps, for example in the norther part of the $\mathrm{PA}$ at $0^{\circ}$ and in the inner region of the PA at $295^{\circ}$ are caused by either an extremely faint, hence not measurable [S II] $\lambda 6731$ line, or a contamination of that line by a strong cosmic ray, or a combination of both.

A few outliers appear with flux ratio values greater than the theoretically predicted one. We checked these flux measurements by eye, and they seem to be appropriate. Therefore, we believe that in these extremely low-density regions our assumption of pure collisional excitation of the levels might not hold anymore.

\subsection{Analysis of the photometric light curve}

The TESS time series of the central object MWC 137 taken in sector 6 and sector 33 are displayed in the top left and right panels of Figure 10, respectively. A slightly higher mean flux value was noted for sector 33, corresponding to a difference of $0.022 \mathrm{mag}$. Considering the two year time gap between the observations, this brightening (if real and not just caused by very slight changes in the aperture and centering between sectors or due to contamination from nearby sources) might be related to a possible long-term variability of $332.4 \mathrm{~d}$. This period has been suggested by Maravelias et al. (2018) based on their analysis of the ground-based light curve provided by the ASAS survey (Pojmanski 1997), covering a total of about $2500 \mathrm{~d}$ but with very sparse coverage. Such a long period cannot be recovered by the current set of TESS data. Therefore, we restrict our analysis to the light variability for each sector independently, considering their individual mean magnitudes. 


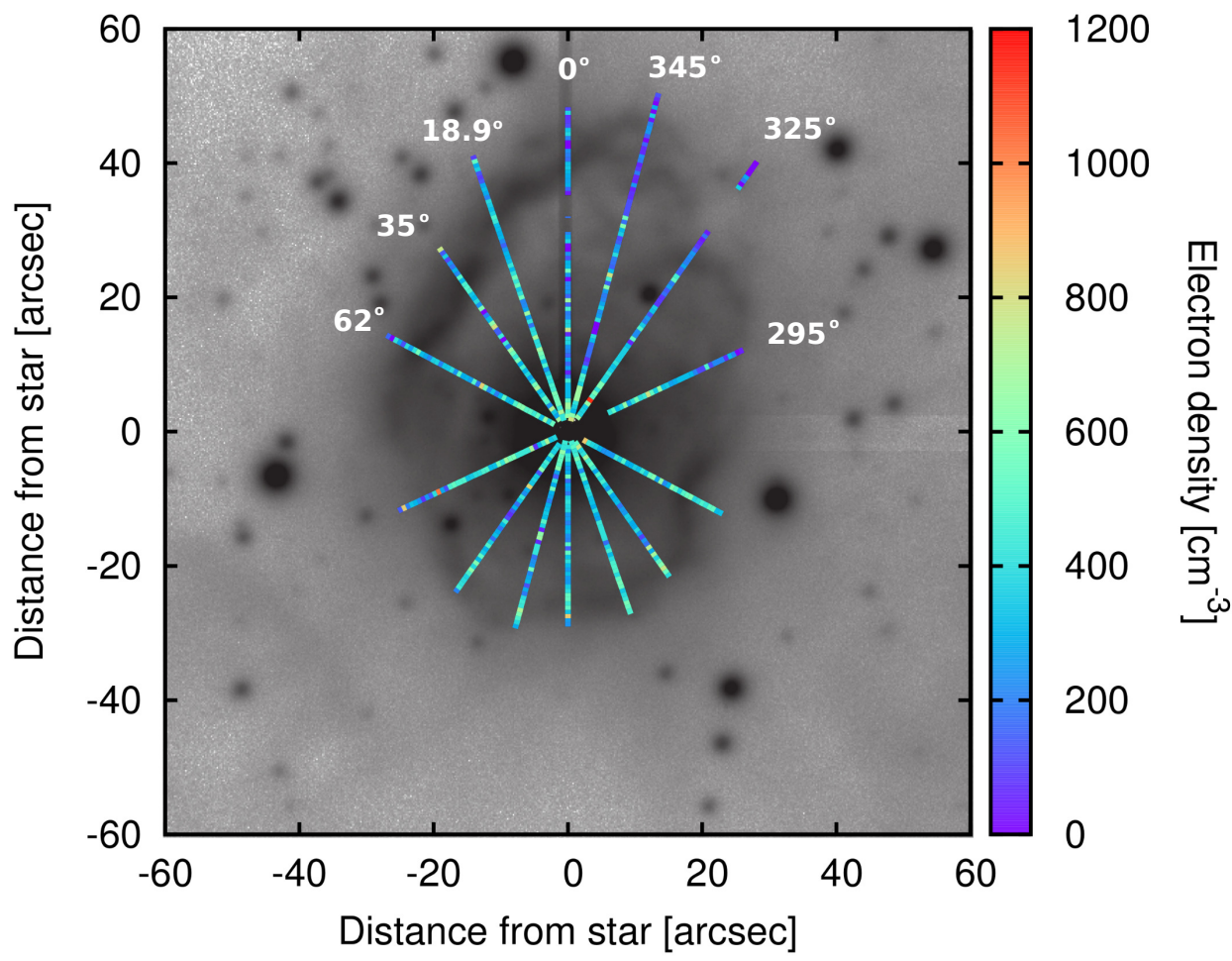

Figure 9. Color presentation of the electron density along the different slits for a constant electron temperature of $10000 \mathrm{~K}$ across the nebula.

The two pieces of the light curve of MWC 137 display clear but complex variability. To search for periodicities in these data sets, we perform a frequency analysis utilizing Period04 (Lenz \& Breger 2005) in a standard pre-whitening procedure. The amplitude spectra of the original data are shown in the lower parts of Figure 10. They have been computed up to the Nyquist frequency $\left(\sim 360 \mathrm{~d}^{-1}\right.$ in both cases) and they display clear differences in the signal strengths, especially in the lowfrequency $\left(<0.5 \mathrm{~d}^{-1}\right)$ domain. No significant peaks are detected beyond $2 \mathrm{~d}^{-1}$. We also include the amplitude spectrum of the combined data set (bottom panel).

The short observational baselines within the TESS sectors lead to several constraints for the identification of significant frequencies. First, it does not allow to measure signals with periods greater than $\sim 10 \mathrm{~d}$, so that we ignore any detections in the low-frequency $\left(\leq 0.1 \mathrm{~d}^{-1}\right)$ range. Second, while the Rayleigh resolution, defined as $1 / T$ where $T$ is the time span of the observations, amounts to $0.046 \mathrm{~d}^{-1}$ and $0.039 \mathrm{~d}^{-1}$ for the data in sector 6 and 33, respectively, high-amplitude side-lobes appear around dominant peaks in the amplitude spectra (see Figure 11). Therefore, we follow the conservative approach proposed by Loumos \& Deeming (1978) and discard frequencies with a separation of less than 2.5 times the Rayleigh resolution to previously identified frequencies with higher amplitude. Third, a higher thresh- old $(S / N \geq 5)$ for the significance of identified peaks is recommended for an observational baseline of less than a few months of space photometry (Baran et al. 2015). And finally, a densely populated amplitude spectrum in the low-frequency domain requires a wider window size of $5 \mathrm{~d}^{-1}$ instead of the conventional $1 \mathrm{~d}^{-1}$ for the computation of the underlying noise spectrum (Burssens et al. 2020).

Applying all these constraints during the frequency analysis, we end up with only a small number of frequencies that we consider as reliable. These frequencies are shown in the amplitude spectra of Figure 10 and listed in Table 2. We clearly identify one dominant signal in both sectors with a period of $1.93 \mathrm{~d}$. The frequencies of its first two harmonics ( $2 \mathrm{~F} 1$ and $3 \mathrm{~F} 1)$ are included in Figure 10. They are both seen in the amplitude spectrum of sector 33, whereas in sector 6 only the first harmonics is evident. Their signals fall slightly below our detection criterion $(4<S / N<5)$ so that we include their frequencies only at the bottom of Table 2. The closeness of frequencies F2 in sector 6 and F4 in sector 33 suggests that they might be the same. If true, then this frequency could be another persistent one, while the other two frequencies (F3, F5) that show high amplitudes in sector 33, are not evident in sector 6 and thus seem to be stochastic. 

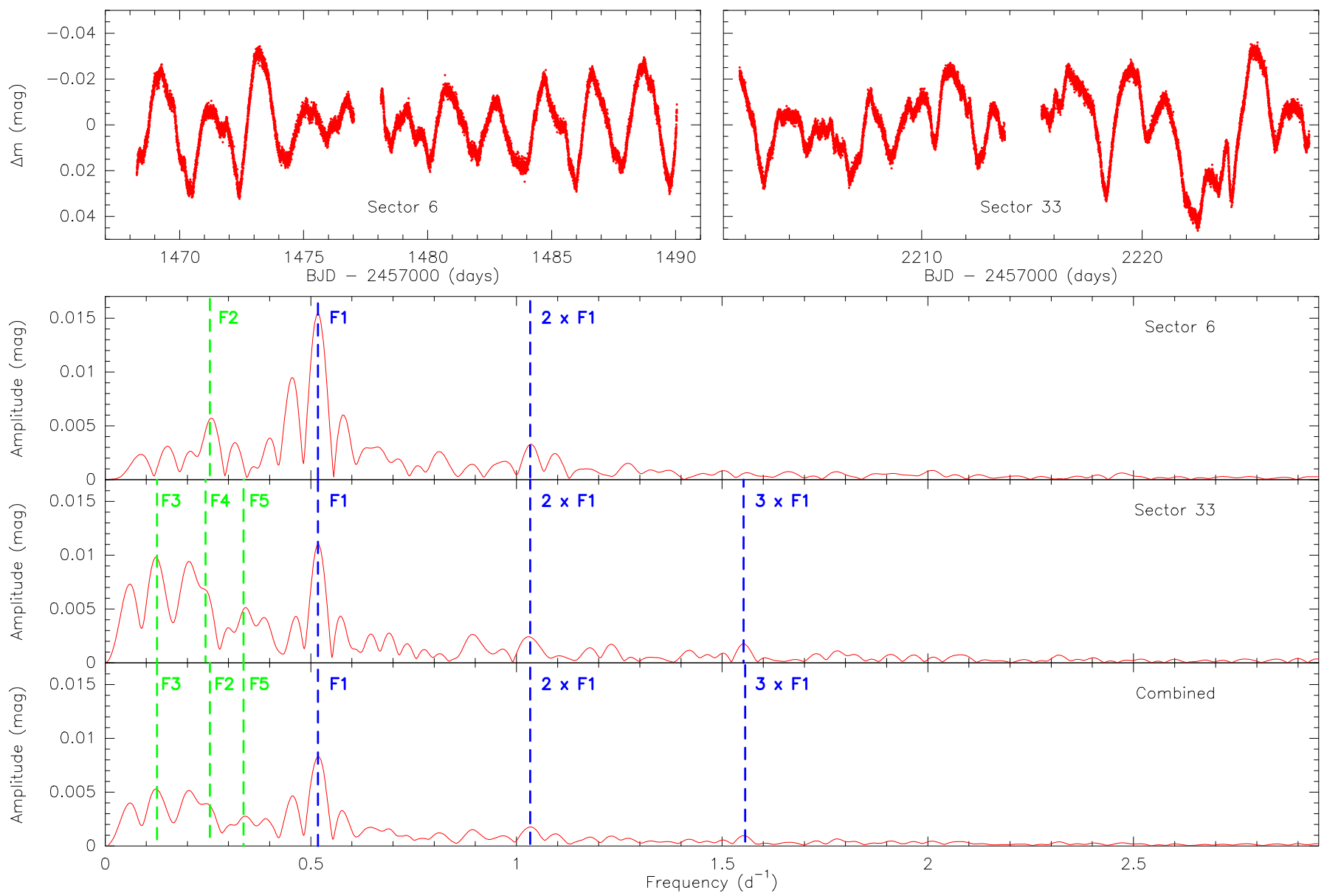

Figure 10. TESS light curve of MWC 137 in the two sectors (top) and their first Fourier transform (bottom). Identified frequencies are indicated.

\section{DISCUSSION}

\subsection{Large-scale nebula}

In a previous study Kraus et al. (2017) proposed a toy model for the nebula of MWC 137, consisting of two interwoven double-cones offset by $5 . .5$ in north-south direction. The northern one was situated and sheared such that it fitted to the large-scale putative doublering structure, whereas the southern one was oriented in a way that its symmetry axis was aligned with the jet axis.

Our new observations provide no complementary evidence that would support this model. Instead, the deeper image in the lines of [S II] imply that the largescale nebula consists of helical or spiral-arm like structures rather than a double-ring (see Figure 2). The RV measurements suggest that these helical structures are blue-shifted in the northern nebular regions and redshifted in the southern ones, whereas the diffuse gas displays complex velocity variations. The lack of information about the velocity of the gas in the plane of the
Table 2. Significant frequencies identified in the TESS light curve. The harmonics of $\mathrm{F} 1$ are listed at the bottom.

\begin{tabular}{lrr}
\hline \hline & $\begin{array}{r}\text { Sector 6 } \\
\text { Frequency } \\
\left(\mathrm{d}^{-1}\right)\end{array}$ & $\begin{array}{r}\text { Sector 33 } \\
\text { Frequency } \\
\left(\mathrm{d}^{-1}\right)\end{array}$ \\
\hline $\mathrm{F} 1$ & 0.51671400 & 0.51865874 \\
$\mathrm{~F} 2$ & 0.25491224 & $\ldots$ \\
$\mathrm{F} 3$ & $\ldots$ & 0.12579410 \\
$\mathrm{~F} 4$ & $\ldots$ & 0.24384702 \\
$\mathrm{~F} 5$ & $\ldots$ & 0.33674112 \\
\hline 2F1 & 1.03572450 & 1.03538218 \\
3F1 & $\ldots$ & 1.55210563 \\
\hline
\end{tabular}

sky clearly hampers to draw conclusions about the real motion of the nebular structures in three dimensional space. But for the detection of the nebular expansion in the plane of the sky, our current baseline of $18.1 \mathrm{yr}$ is still too short. 

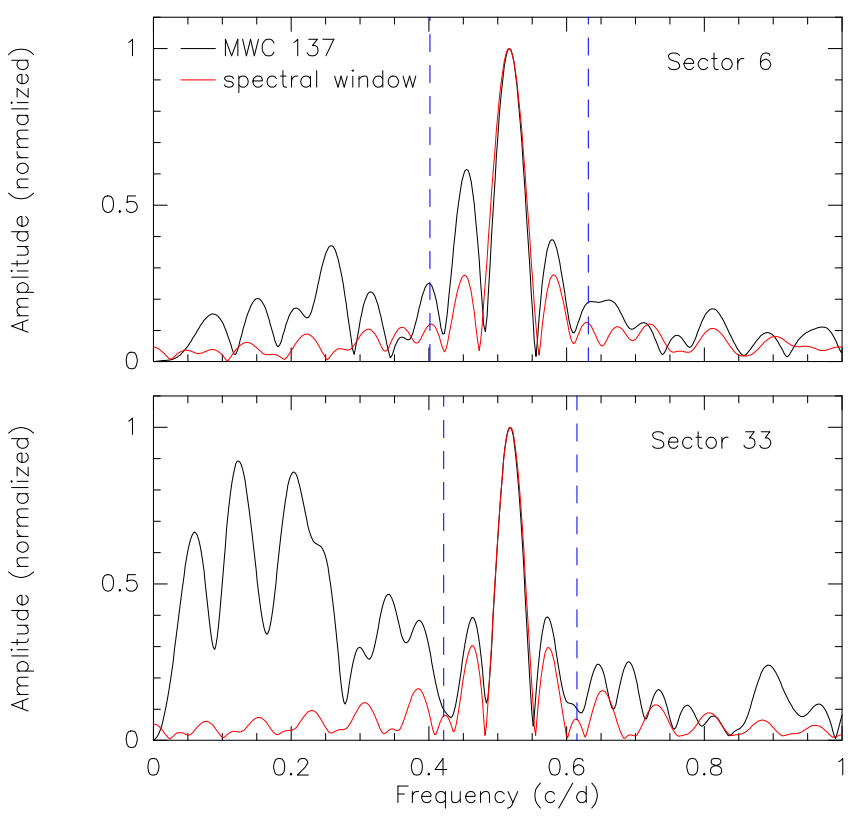

Figure 11. Spectral window (red) for the two sectors centered on the frequency of the dominant peak in our original data (black). Also shown is the frequency resolution defined as 2.5 times the Rayleigh resolution (blue dashed lines).

\subsection{Stellar mass and age}

The significantly improved distance estimate towards MWC 137 based on Gaia, which agrees very well with the value derived by Mehner et al. (2016), allows us to finally settle the luminosity of the star and to better constrain its initial and current mass. The huge range of previously proposed distances resulted in luminosity estimates spreading from $\log \left(L / L_{\odot}\right)=4.18$ (AlonsoAlbi et al. 2009) up to at least 5.37 (Esteban \& Fernandez 1998), and thus in masses ranging from 12 to at least $32 M_{\odot}$ when compared to stellar evolutionary tracks of rotating and non-rotating stars (see Muratore et al. 2015). The most recent mass estimate has been performed by Mehner et al. (2016), who fit the location of MWC 137 in a color-magnitude diagram with GENEVA isochrones for the cluster and evolutionary tracks from Ekström et al. (2012) based on which they claim best agreement for an age of $\sim 10 \mathrm{Myr}$ and a mass of $\sim 15 M_{\odot}$. At the same time, they estimate the luminosity of MWC 137 as $\log \left(L / L_{\odot}\right) \sim 6$, which clearly contradicts the low mass assigned to the object.

We believe that this mismatch between mass and luminosity arises from the fact that Mehner et al. (2016) only matched the models with the absolute visual magnitude, $M_{V}$, but they neglected the effective temperature of the star. Temperature estimates in the literature all point towards a hot central object of $T_{\text {eff }}=$ $25000-31000 \mathrm{~K}$ (see, e.g., Hillenbrand et al. 1992; Es- teban \& Fernandez 1998; Alonso-Albi et al. 2009). Using the values of Mehner et al. (2016) for the visual magnitude $V$ and the extinction $A_{V}$, along with the Gaia distance (Table 3), the absolute visual magnitude of MWC 137 is $M_{V}=-7.2 \pm 0.3 \mathrm{mag}$, in excellent agreement with the value of Mehner et al. (2016).

In Figure 12 we display evolutionary tracks for nonrotating (left panel) and rotating stellar models with $\Omega_{\text {in }} / \Omega_{\text {crit }}=0.568$ (respectively $v / v_{\text {crit }}=0.4$, middle panel) from Ekström et al. (2012) for solar metallicity. If MWC 137 had an initial mass of $15 M_{\odot}$ as proposed by Mehner et al. (2016), a value of $M_{V}=-7.2 \mathrm{mag}$ is achieved only when the star has reached an effective temperature $\log T_{\text {eff }} \leq 4$ according to the models of both rotating and non-rotating stars. Considering the proper effective temperature range for $\mathrm{MWC} 137$ of $\log T_{\text {eff }}=$ $4.40-4.49$, the real initial mass is considerably higher than $15 M_{\odot}$, spreading from about 35 to $65 M_{\odot}$.

To further narrow down the mass range, we included in Figure 12 information about the surface abundance ratio of ${ }^{13} \mathrm{C} /{ }^{12} \mathrm{C}$. This ratio is very sensitive to stellar rotation and may help to make inferences about the initial rotation speed of MWC 137 . While we cannot measure the carbon isotope abundance ratio directly on the stellar surface, Kraus (2009) has shown that the enrichment of the close-by circumstellar environment in the isotopic molecule ${ }^{13} \mathrm{CO}$ provides a reasonable tracer of the stellar surface enrichment in ${ }^{13} \mathrm{C}$ for stars with strong winds or mass ejections. The circumstellar matter of MWC 137 indicates a carbon isotope abundance ratio of ${ }^{13} \mathrm{C} /{ }^{12} \mathrm{C}=25 \pm 2$ (Muratore et al. 2015; Oksala et al. 2013). While the non-rotating tracks do not display significant surface enrichment at early stellar evolutionary phases, the models with rotation predict that the amount observed in MWC 137 is reached already during the main sequence and significantly lower around the position of MWC 137 in the HR diagram. Therefore, to achieve the observed value, the initial rotation rate of MWC 137 must be clearly non-zero but also lower than $\Omega_{\text {in }} / \Omega_{\text {crit }}=0.568$.

We interpolate the pre-calculated evolution tracks of Ekström et al. (2012) using their tool SYCLIST ${ }^{4}$ to obtain stellar evolution tracks for a dense mass grid and covering rotation rates between $\Omega_{\text {in }} / \Omega_{\text {crit }}=0$ and 0.568 . The evolution of the carbon isotope ratio is followed along these tracks to select the best fitting models for the parameters of MWC 137. We find, that the most likely initial mass of MWC 137 is $49 \pm 11 M_{\odot}$ with an initial

\footnotetext{
${ }^{4}$ https://www.unige.ch/sciences/astro/evolution/en/database/ syclist/
} 
Table 3. Stellar parameters of MWC 137.

\begin{tabular}{cccccccccccc}
\hline \hline $\begin{array}{c}V \\
(\mathrm{mag})\end{array}$ & $\begin{array}{c}E(B-V) \\
(\mathrm{mag})\end{array}$ & $\begin{array}{c}A_{V} \\
(\mathrm{mag})\end{array}$ & $\begin{array}{c}d \\
(\mathrm{kpc})\end{array}$ & $\begin{array}{c}M_{V} \\
(\mathrm{mag})\end{array}$ & $\begin{array}{c}\log T_{\text {eff }} \\
(\mathrm{K})\end{array}$ & $\log L / L_{\odot}$ & $M_{\text {in }}$ & $M$ & $R$ & $\Omega_{\text {in }} / \Omega_{\text {crit }}$ & Age \\
$\left(M_{\odot}\right)$ & $\left(M_{\odot}\right)$ & $\left(R_{\odot}\right)$ & & $(\mathrm{Myr})$ \\
\hline $11.95^{a}$ & $1.8^{a}$ & $5.6^{a}$ & $5.15_{-0.60}^{+0.79}$ & $-7.2 \pm 0.3$ & $4.40-4.49^{b}$ & $5.84 \pm 0.13$ & $49 \pm 11$ & $37_{-5}^{+9}$ & $25_{-9}^{+20}$ & $0.425 \pm 0.025$ & $4.7 \pm 0.8$ \\
\hline
\end{tabular}

References_ ${ }^{a}$ Mehner et al. (2016); ${ }^{b}$ Hillenbrand et al. (1992); Esteban \& Fernandez (1998); Alonso-Albi et al. (2009).
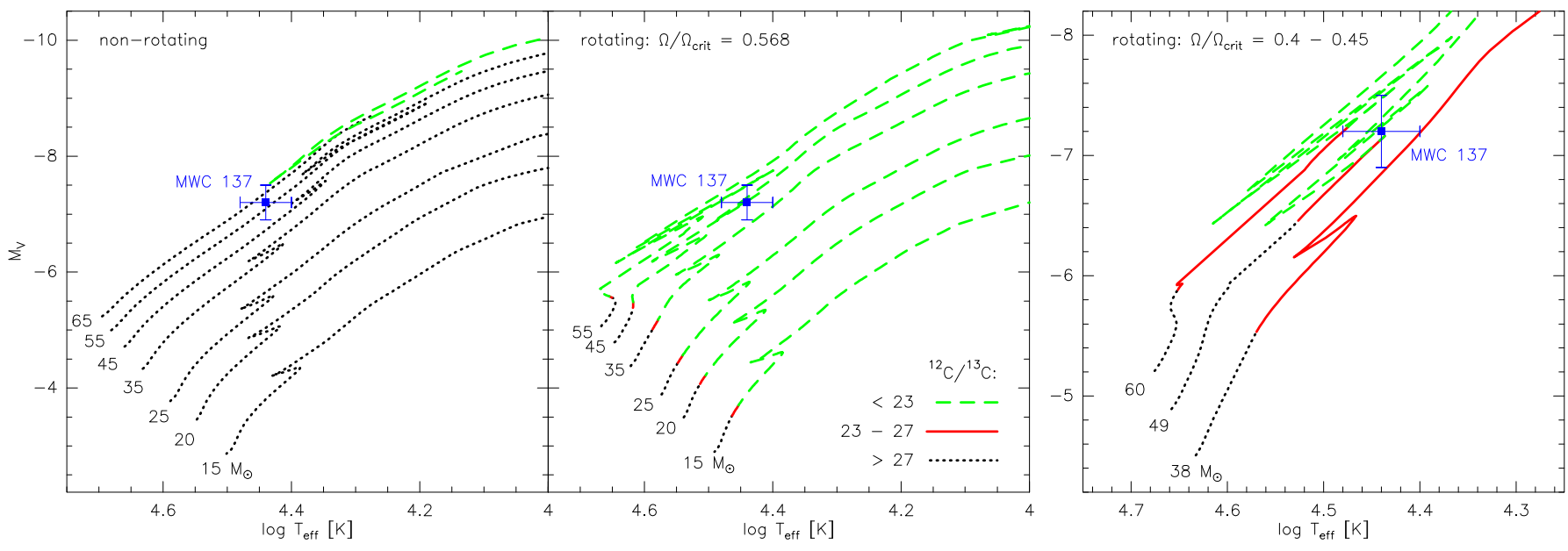

Figure 12. Position of MWC 137 in the HR diagram. Evolutionary tracks for non-rotating (left) and rotating (middle) stellar models at solar metallicity are from Ekström et al. (2012). The color coding of the tracks refers to different ranges of the carbon isotope abundance ratio on the stellar surface with the solid red line covering the measurements for MWC 137. The right panel shows a zoomed version and evolutionary tracks for rotation speeds that predict the proper carbon isotope ratio at the position of MWC 137.

rotation rate of $\Omega_{\text {in }} / \Omega_{\text {crit }}=0.40-0.43$. Slightly higher rotation rate values (up to 0.45 ) are still possible for models at the upper mass limit. Representative tracks are shown in the right panel of Figure 12. From these tracks we deduce a current stellar mass of $37_{-5}^{+9} M_{\odot}$ and a luminosity of $\log \left(L / L_{\odot}\right)=5.84 \pm 0.13$ for MWC 137 . The latter is in good agreement with the luminosity of $\log \left(L / L_{\odot}\right) \sim 6$ derived by Mehner et al. (2016). The final set of stellar parameters of MWC 137 is presented in Table 3. The table also includes the plausible age of MWC 137, which is found to range from 3.9 to $5.5 \mathrm{Myr}$, in excellent agreement with the estimated ages of the other stars in that cluster (see Mehner et al. 2016).

\subsection{Photometric variability}

The TESS time series, taken in sectors 6 and 33 with a separation of two years, contains at least one stable period of $1.93 \mathrm{~d}$ (see Sect.3.5). Its putative decrease in amplitude between the two observing runs (see Figure 10) might not be real but caused by the simultaneous weakening or disappearance of possible, unresolved stochastic signals in the vicinity of the dominant frequency. Considering the stellar parameters of MWC 137 (see Table 3), it is obvious that a period of $1.93 \mathrm{~d}$ is way too short to be interpreted as the rotation velocity of the star. The eligible stellar models predict an equatorial rotation velocity of $<10 \mathrm{~km} \mathrm{~s}^{-1}$ for the current evolutionary state of MWC 137, resulting in a minimum stellar rotation period of $\sim 100 \mathrm{~d}$. It is also unlikely that the $1.93 \mathrm{~d}$ period could be caused by the orbital motion of a hidden companion. Considering the parameter range for MWC 137, the corresponding orbital radius of the companion would lie inside the stellar radius. Therefore, a different alternative for the appearance of the $1.93 \mathrm{~d}$ period seems to be more likely, and this is stellar pulsation.

The other, stochastic or random signals seen in the amplitude spectrum of MWC 137 might be related to internal gravity waves. The appearance of such waves has been suggested for early-type stars, and they seem to be suitable to generate stochastic photometric variability (Rogers et al. 2013). The clearly variable amplitude and evanescence of these signals further support such an interpretation.

But other or additional mechanisms might be at work and are worth being mentioned. As a massive, early- 
type star MWC 137 has a line-driven wind. Recent investigations have shown that perturbations in the velocity field at the base of the wind, for example by stochastic waves, can cause wind instabilities (Krtička \& Feldmeier 2021). These instabilities can generate variability of the mass flux and hence wind inhomogeneities that might lead to measurable (quasi-periodic or stochastic) changes in photometry. A time variable wind of MWC 137 is evident from moderate changes in its hydrogen Balmer line emission at optical wavelengths (Zickgraf 2003; Kraus et al. 2017) as well as from variable Pfund line emission in the near-infrared (Muratore et al. 2015). Furthermore, the central star is surrounded by a compact region of molecular and neutral gas, possibly arranged in a series of rings (Maravelias et al. 2018), and the absence of photometric lines in the spectra implies that the star is embedded in a dense envelope. A compact disk has also been proposed to be the source of the emanating jet and blobs discovered by Mehner et al. (2016), and dynamical dusty blobs and arc-like structures have been found in the close vicinity of MWC 137 as well (Kraus et al. 2017). Therefore, it is clear that any time-variable structures around the central star can easily imprint variabilities on the observed photometric signals.

\section{CONCLUSIONS}

In this work, we present new optical observations of the large-scale nebula connected to the $\mathrm{B}[\mathrm{e}]$ supergiant star MWC 137. We find no evidence for an expansion of the nebular structures in the plane of the sky based on $\mathrm{H} \alpha$ images spanning over more than $18 \mathrm{yr}$. This means that the tangential velocity must be smaller than $\sim 53 \mathrm{~km} \mathrm{~s}^{-1}$ for the distance of $5.15 \mathrm{kpc}$ to MWC 137 . The RVs measured from the [N II] and [S II] emission lines reveal a complex pattern across the nebula. But in general, the data confirm the previous findings that the northern nebular regions are predominantly approaching whereas the southern ones are mostly receding. We also find that the velocity dispersion is highest in regions of highest RV. Moreover, the RV measurements reveal systematically higher values for the [N II] lines in the blueshifted north and north-western regions as well as in the red-shifted south-eastern area in which the RVs are highest. These regions coincide with the regions of lowest electron densities. The clear correlation of higher RV with higher ionization potential of the element suggests a velocity stratification along the line of sight, meaning that we observe a decrease in RV from inside out. Whether this decrease is consistent with ballistic expansion needs to be checked once tangential velocities have been measured. Proper fully 3D kinematics will also help to understand the formation scenario of the diverse observed spiral-arm like structures.

In addition to the spectroscopic data, we also investigate the photometric light curve provided by the TESS space mission. Our analysis reveals a few low-frequency stochastic or random signals and one stable $1.93 \mathrm{~d}$ period over the observing baseline of $2 \mathrm{yr}$. This period can neither be related to the rotation velocity of the star, which is minimum $100 \mathrm{~d}$ considering our derived mass of $\sim 37 \mathrm{M}_{\odot}$, luminosity of $\log L / L_{\odot} \sim 5.8$, and age of $3.9-5.5$ Myr of MWC 137, nor can it be interpreted as orbital motion of a hidden companion. Therefore, stellar pulsation might be a plausible alternative. The real nature of both the $1.93 \mathrm{~d}$ and the low-frequency stochastic variability certainly requires further investigation, including computations of stellar pulsation models for the parameter space of MWC 137. However, this is beyond the scope of the present work.

\section{ACKNOWLEDGMENTS}

This research made use of the NASA Astrophysics Data System (ADS), of the SIMBAD database, operated at CDS, Strasbourg, France, and of data from the European Space Agency (ESA) mission Gaia (https://www. cosmos.esa.int/gaia), processed by the Gaia Data Processing and Analysis Consortium (DPAC, https://www. cosmos.esa.int/web/gaia/dpac/consortium). Funding for the DPAC has been provided by national institutions, in particular the institutions participating in the Gaia Multilateral Agreement. Parts of the data presented here were obtained with ALFOSC, which is provided by the Instituto de Astrofisica de Andalucia (IAA) under a joint agreement with the University of Copenhagen and NOTSA, and with the 6-m telescope of the Special Astrophysical Observatory, operated with the financial support of the Ministry of Education and Science of the Russian Federation (agreement No. 14.619.21.0004, project ID RFMEFI61914X0004). This paper includes also data collected with the TESS mission, obtained from the MAST data archive at the Space Telescope Science Institute (STScI). Funding for the TESS mission is provided by the NASA Explorer Program. STScI is operated by the Association of Universities for Research in Astronomy, Inc., under NASA contract NAS 5-26555.

We wish to thank Miguel Santander-García for advice on using the magnification method, and Anthony Marston for providing us with his $\mathrm{H} \alpha$ image. MK, JPSA, and DHN acknowledge financial support from the Czech Science Foundation (GA ČR 20-00150S). The Astronomical Institute Ondřejov is supported by the 
project RVO:67985815. LSC acknowledges financial support from CONICET (PIP 0177) and from the Agencia Nacional de Promoción Científica y Tecnológica de Argentina (Préstamo BID PICT 2016-1971) DJ acknowledges support from the Erasmus+ programme of the European Union under grant number 2020-1-CZ01KA203-078200. This project has received funding from the European Union's Framework Programme for Re- search and Innovation Horizon 2020 (2014-2020) under the Marie Skłodowska-Curie Grant Agreement No. 823734 .

\section{Facility: NOT}

Facility: BAT

Facility: Danish $1.54 \mathrm{~m}$ Telescope

\section{REFERENCES}

Ababakr, K. M., Oudmaijer, R. D., \& Vink, J. S. 2017, MNRAS, 472, 854, doi: 10.1093/mnras/stx1891

Afanasiev, V. L., \& Moiseev, A. V. 2011, Baltic Astronomy, 20, 363, doi: 10.1515/astro-2017-0305

Allen, D. A., \& Glass, I. S. 1976, ApJ, 210, 666, doi: $10.1086 / 154872$

Alonso-Albi, T., Fuente, A., Bachiller, R., et al. 2009, A\&A, 497, 117, doi: 10.1051/0004-6361/200810401

Arellano-Córdova, K. Z., Esteban, C., García-Rojas, J., \& Méndez-Delgado, J. E. 2020, MNRAS, 496, 1051, doi: 10.1093/mnras/staa1523

Aret, A., Kraus, M., Muratore, M. F., \& Borges Fernandes, M. 2012, MNRAS, 423, 284, doi: 10.1111/j.1365-2966.2012.20871.x

Arun, R., Mathew, B., Manoj, P., et al. 2019, AJ, 157, 159, doi: 10.3847/1538-3881/ab0ca1

Baran, A. S., Koen, C., \& Pokrzywka, B. 2015, MNRAS, 448, L16, doi: 10.1093/mnrasl/slu194

Berrilli, F., Corciulo, G., Ingrosso, G., et al. 1992, ApJ, 398, 254, doi: 10.1086/171853

Bonanos, A. Z., Massa, D. L., Sewilo, M., et al. 2009, AJ, 138, 1003, doi: 10.1088/0004-6256/138/4/1003

Bonanos, A. Z., Lennon, D. J., Köhlinger, F., et al. 2010, AJ, 140, 416, doi: 10.1088/0004-6256/140/2/416

Brandner, W., Grebel, E. K., Chu, Y.-H., \& Weis, K. 1997, ApJL, 475, L45, doi: 10.1086/310460

Burssens, S., Simón-Díaz, S., Bowman, D. M., et al. 2020, A\&A, 639, A81, doi: 10.1051/0004-6361/202037700

Caicedo-Ortiz, H. E., \& Fernandez, H. O. C. 2019, Astrophysics, 62, 57, doi: 10.1007/s10511-019-09564-9

Cidale, L. S., Borges Fernandes, M., Andruchow, I., et al. 2012, A\&A, 548, A72, doi: 10.1051/0004-6361/201220120

Cochetti, Y. R., Kraus, M., Arias, M. L., et al. 2020, AJ, 160, 166, doi: 10.3847/1538-3881/abae62

Ekström, S., Georgy, C., Eggenberger, P., et al. 2012, A\&A, 537, A146, doi: 10.1051/0004-6361/201117751

Esteban, C., \& Fernandez, M. 1998, MNRAS, 298, 185, doi: 10.1046/j.1365-8711.1998.01610.x

Esteban, C., \& Peimbert, M. 1999, A\&A, 349, 276
Fich, M., \& Blitz, L. 1984, ApJ, 279, 125, doi: $10.1086 / 161872$

Fuente, A., Rodríguez-Franco, A., Testi, L., et al. 2003, ApJL, 598, L39, doi: 10.1086/380296

Gaia Collaboration, Prusti, T., de Bruijne, J. H. J., et al. 2016, A\&A, 595, A1, doi: 10.1051/0004-6361/201629272

Gaia Collaboration, Brown, A. G. A., Vallenari, A., et al. 2021, A\&A, 649, A1, doi: 10.1051/0004-6361/202039657

Hillenbrand, L. A., Strom, S. E., Vrba, F. J., \& Keene, J. 1992, ApJ, 397, 613, doi: 10.1086/171819

Kraus, M. 2009, A\&A, 494, 253, doi: 10.1051/0004-6361:200811020

—. 2019, Galaxies, 7, 83, doi: 10.3390/galaxies7040083

Kraus, M., Arias, M. L., Cidale, L. S., \& Torres, A. F. 2020, MNRAS, 493, 4308, doi: 10.1093/mnras/staa519

Kraus, M., Borges Fernandes, M., \& de Araújo, F. X. 2007, A\&A, 463, 627, doi: 10.1051/0004-6361:20066325

-. 2010, A\&A, 517, A30, doi: 10.1051/0004-6361/200913964

Kraus, M., Cidale, L. S., Arias, M. L., Oksala, M. E., \& Borges Fernandes, M. 2014, ApJL, 780, L10, doi: 10.1088/2041-8205/780/1/L10

Kraus, M., Oksala, M. E., Cidale, L. S., et al. 2015, ApJL, 800, L20, doi: 10.1088/2041-8205/800/2/L20

Kraus, M., Cidale, L. S., Arias, M. L., et al. 2016, A\&A, 593, A112, doi: 10.1051/0004-6361/201628493

Kraus, M., Liimets, T., Cappa, C. E., et al. 2017, AJ, 154, 186, doi: 10.3847/1538-3881/aa8df6

Krtička, J., \& Feldmeier, A. 2021, A\&A, 648, A79, doi: 10.1051/0004-6361/202040148

Lamers, H. J. G. L. M., Zickgraf, F.-J., de Winter, D., Houziaux, L., \& Zorec, J. 1998, A\&A, 340, 117

Lenz, P., \& Breger, M. 2005, Communications in Asteroseismology, 146, 53, doi: 10.1553/cia146s53

Liermann, A., Kraus, M., Schnurr, O., \& Fernandes, M. B. 2010, MNRAS, 408, L6, doi: 10.1111/j.1745-3933.2010.00915.x

Liermann, A., Schnurr, O., Kraus, M., et al. 2014, MNRAS, 443, 947, doi: 10.1093/mnras/stu1174 
Liimets, T., Corradi, R. L. M., Jones, D., et al. 2018, A\&A, 612, A118, doi: 10.1051/0004-6361/201732073

Loumos, G. L., \& Deeming, T. J. 1978, Ap\&SS, 56, 285, doi: $10.1007 / \mathrm{BF} 01879560$

Luri, X., Brown, A. G. A., Sarro, L. M., et al. 2018, A\&A, 616, A9, doi: 10.1051/0004-6361/201832964

Maravelias, G., Kraus, M., Cidale, L. S., et al. 2018, MNRAS, 480, 320, doi: 10.1093/mnras/sty 1747

Marston, A. P., \& McCollum, B. 2008, A\&A, 477, 193, doi: 10.1051/0004-6361:20066086

McGregor, P. J., Hyland, A. R., \& Hillier, D. J. 1988, ApJ, 334, 639, doi: 10.1086/166867

Mehner, A., de Wit, W. J., Groh, J. H., et al. 2016, A\&A, 585, A81, doi: 10.1051/0004-6361/201527180

Moiseev, A. V. 2002, Bulletin of the Special Astrophysics Observatory, 54, 74 .

https://arxiv.org/abs/astro-ph/0211104

—. 2015, Astrophysical Bulletin, 70, 494, doi: 10.1134/S1990341315040112

Moiseev, A. V., \& Egorov, O. V. 2008, Astrophysical Bulletin, 63, 181, doi: 10.1134/S1990341308020089

Morris, P. W., Eenens, P. R. J., Hanson, M. M., Conti, P. S., \& Blum, R. D. 1996, ApJ, 470, 597, doi: 10.1086/177892

Muratore, M. F., Kraus, M., Oksala, M. E., et al. 2015, AJ, 149, 13, doi: 10.1088/0004-6256/149/1/13

Oksala, M. E., Kraus, M., Arias, M. L., et al. 2012, MNRAS, 426, L56, doi: 10.1111/j.1745-3933.2012.01323.x

Oksala, M. E., Kraus, M., Cidale, L. S., Muratore, M. F., \& Borges Fernandes, M. 2013, A\&A, 558, A17, doi: 10.1051/0004-6361/201321568

Oudmaijer, R. D., \& Drew, J. E. 1999, MNRAS, 305, 166, doi: 10.1046/j.1365-8711.1999.02383.x
Pojmanski, G. 1997, AcA, 47, 467. https://arxiv.org/abs/astro-ph/9712146

Reed, D. S., Balick, B., Hajian, A. R., et al. 1999, AJ, 118, 2430, doi: 10.1086/301091

Rogers, T. M., Lin, D. N. C., McElwaine, J. N., \& Lau, H. H. B. 2013, ApJ, 772, 21, doi: 10.1088/0004-637X/772/1/21

Sandell, G., Weintraub, D. A., \& Hamidouche, M. 2011, ApJ, 727, 26, doi: 10.1088/0004-637X/727/1/26

Smith, N., Bally, J., \& Walawender, J. 2007, AJ, 134, 846, doi: $10.1086 / 518563$

Tayal, S. S., \& Zatsarinny, O. 2010, ApJS, 188, 32, doi: 10.1088/0067-0049/188/1/32

The, P. S., de Winter, D., \& Perez, M. R. 1994, A\&AS, 104

Torres, A. F., Cidale, L. S., Kraus, M., et al. 2018, A\&A, 612, A113, doi: 10.1051/0004-6361/201731723

Vink, J. S., Drew, J. E., Harries, T. J., \& Oudmaijer, R. D. 2002, MNRAS, 337, 356, doi: 10.1046/j.1365-8711.2002.05920.x

Vioque, M., Oudmaijer, R. D., Baines, D., Mendigutía, I., \& Pérez-Martínez, R. 2018, A\&A, 620, A128, doi: 10.1051/0004-6361/201832870

Wheelwright, H. E., de Wit, W. J., Weigelt, G., Oudmaijer, R. D., \& Ilee, J. D. 2012, A\&A, 543, A77, doi: 10.1051/0004-6361/201219325

Wouterloot, J. G. A., Brand, J., \& Henkel, C. 1988, A\&A, 191,323

Zickgraf, F.-J. 2003, A\&A, 408, 257, doi: 10.1051/0004-6361:20030999

Zickgraf, F. J., Wolf, B., Stahl, O., Leitherer, C., \& Appenzeller, I. 1986, A\&A, 163, 119

Zickgraf, F. J., Wolf, B., Stahl, O., Leitherer, C., \& Klare, G. 1985, A\&A, 143, 421 